\title{
Low-energy probes of sterile neutrino transition magnetic moments
}

\author{
O.G. Miranda, ${ }^{a}$ D.K. Papoulias, ${ }^{b}$ O. Sanders, ${ }^{a}$ M. Tórtola ${ }^{c, d}$ and J.W.F. Valle ${ }^{d}$ \\ ${ }^{a}$ Departamento de Física, Centro de Investigación y de Estudios Avanzados del IPN, \\ Apartado Postal 14-740, Distrito Federal 07000, Mexico \\ ${ }^{b}$ Department of Physics, University of Ioannina, \\ University Campus, Ioannina GR 45110, Greece \\ ${ }^{c}$ Departament de Fúsica Teórica, Universitat de València, \\ Dr. Moliner 50, Burjassot 46100, Spain \\ ${ }^{d}$ AHEP Group, Institut de Física Corpuscular, CSIC/Universitat de València, \\ C/ Catedrático José Beltrán, 2, Paterna E-46980, Spain \\ E-mail: omr@fis.cinvestav.mx, d.papoulias@uoi.gr, \\ osanders@fis.cinvestav.mx, mariam@ific.uv.es, valle@ific.uv.es
}

Abstract: Sterile neutrinos with keV-MeV masses and non-zero transition magnetic moments can be probed through low-energy nuclear or electron recoil measurements. Here we determine the sensitivities of current and future searches, showing how they can probe a previously unexplored parameter region. Future coherent elastic neutrino-nucleus scattering $(\mathrm{CE} \nu \mathrm{NS})$ or elastic neutrino-electron scattering $(\mathrm{E} \nu \mathrm{ES})$ experiments using a monochromatic ${ }^{51} \mathrm{Cr}$ source can fully probe the region indicated by the recent XENON1T excess.

Keywords: Beyond Standard Model, CP violation, Neutrino Physics, Solar and Atmospheric Neutrinos

ARXiv EPrint: 2109.09545 


\section{Contents}

1 Introduction 1

2 Basic formalism 2

2.1 Neutrino magnetic moment and massive "sterile" leptons 2

2.2 $\mathrm{CE} \nu \mathrm{NS}$ and neutrino-electron scattering in the SM 6

2.3 Sterile neutrino dipole portal 8

2.4 Event rates 9

3 Experimental tests of neutrino magnetic moments 9

$\begin{array}{lll}3.1 & \text { XENON1T } & 10\end{array}$

$\begin{array}{lll}3.2 & \text { COHERENT } & 10\end{array}$

3.3 TEXONO 11

$3.4 \mathrm{CE} \nu \mathrm{NS}$ and $\mathrm{E} \nu \mathrm{ES}$ with a ${ }^{51} \mathrm{Cr}$ source 12

4 Results 13

5 Conclusions and outlook $\quad 17$

\section{Introduction}

Despite their poor theoretical motivation, the existence of light electroweak singlet sterile neutrinos may point to a radical departure from the Standard Model (SM), such as extra space-time dimensions [1-3], the existence of new protecting symmetries, such as a nearly conserved lepton number [4, 5], or the presence of a singular seesaw mechanism [6], amongst other possibilities. From this viewpoint, probing the existence of light sterile neutrinos is extremely interesting. Depending on the mass and mixing parameters characterizing the light sterile states, there can be stringent restrictions following from laboratory, astrophysical and cosmological observations [7-10].

An attractive theoretical benchmark employing the type-I seesaw mechanism based upon the SM gauge symmetry [11] assumes the existence of heavy singlet "right-handed" neutrinos. The existence of such sterile states at the $\mathrm{keV}-\mathrm{MeV}$ mass range [12] would provide a very interesting cosmological scenario in which the oscillations of two heavier singlet right-handed neutrinos of the seesaw mechanism produce lepton number asymmetries. These, in turn, induce a successful leptogenesis picture that account for the observed baryon asymmetry of the Universe [13], while the light right-handed neutrino accounts for cosmological dark matter [14]. Recently, interesting variant scenarios have been discussed, see, e.g. [15].

In this paper, we examine the electromagnetic properties of Majorana neutrinos [1620]. These would imply, for example, the existence of a solar antineutrino flux. Analyzing 
solar neutrino data from the KamLAND experiment, one gets constraints on the Majorana neutrino transition magnetic moments (TMMs) and solar magnetic fields [21, 22]. Likewise, it could account [23] for the excess of electronic recoil events observed in XENON1T [24]. Here we focus on transition magnetic moments in the presence of light electroweak singlet neutrinos. As a motivation, these would have an impact on experiments such as XENON1T [25-27] and IceCube [28]. Neutrino experiments looking for coherent elastic neutrino-nucleus scattering $(\mathrm{CE} \nu \mathrm{NS})$ or elastic neutrino-electron scattering (E $\nu \mathrm{ES})$ events have been proven to be a valuable tool for investigating neutrino oscillations beyond the standard three-neutrino picture, such as deviations from lepton unitarity [29-31], or the presence of light sterile neutrinos [32]. Extending our previous works, here we examine various ways to probe sterile neutrino TMMs through nuclear and electron recoils in various experiments.

For definiteness and simplicity, we assume just one light sterile neutrino. In this case, the neutrino mass matrix and the transition magnetic moments are described by $4 \times 4$ symmetric and anti-symmetric matrices, respectively. The basic $\mathrm{CE} \nu \mathrm{NS}$ and neutrinoelectron scattering cross-sections in the SM are taken from [33, 34]. We discuss current experiments such as COHERENT [35, 36], TEXONO [37] and XENON1T [24], as well as the potential of future $\mathrm{CE} \nu \mathrm{NS}$ and $\mathrm{E} \nu \mathrm{ES}$ experiments using a ${ }^{51} \mathrm{Cr}$ monochromatic neutrino source $[38,39]$.

The paper is organized as follows. The basic formalism describing neutrino magnetic moments in the presence of massive singlet leptons is given in section 2. Section 3 describes various experimental ways of probing neutrino magnetic moments, several of which are new proposals. Our estimated experimental sensitivities are presented in section 4, and a final summary and outlook is provided in section 5 .

\section{Basic formalism}

\subsection{Neutrino magnetic moment and massive "sterile" leptons}

For the case of Majorana particles, the electromagnetic interaction Hamiltonian in the mass basis is given by [16]

$$
H_{e m}^{M}=-\frac{1}{4} \nu_{L}^{T} C^{-1} \tilde{\lambda} \sigma^{\alpha \beta} \nu_{L} F_{\alpha \beta}+\text { h.c. },
$$

where $\tilde{\lambda}$ is the complex antisymmetric matrix describing transition moments, and contains the information on the magnetic and electric dipole moments, $\tilde{\lambda}=\mu-i d$.

In this work, we will assume that neutrinos are Majorana fermions, and we will encode the magnetic and dipole moment information in the aforementioned parameter, $\tilde{\lambda}$. We will also assume that, in addition to the three light active neutrino states, we have extra mass eigenstates associated to gauge singlets. For definiteness, we assume only one of such "sterile" states, so the matrices describing the electromagnetic interaction will be $4 \times 4$,

$$
\tilde{\lambda}=\left(\begin{array}{cccc}
0 & \tilde{\lambda}_{12} & \tilde{\lambda}_{13} & \tilde{\lambda}_{14} \\
-\tilde{\lambda}_{12} & 0 & \tilde{\lambda}_{23} & \tilde{\lambda}_{24} \\
-\tilde{\lambda}_{13} & -\tilde{\lambda}_{23} & 0 & \tilde{\lambda}_{34} \\
-\tilde{\lambda}_{14} & -\tilde{\lambda}_{24} & -\tilde{\lambda}_{34} & 0
\end{array}\right)
$$


Electromagnetic interactions between the (mainly) active to the (mainly) sterile massive states are described by the last row (column) of the matrix, while the flavor transition moments correspond to the upper-left $3 \times 3$ sub-block. ${ }^{1}$ Note that the entries $\tilde{\lambda}_{i j}$ of the transition magnetic moment matrix shown in eq. (2.2) are complex numbers, parametrized in terms of their moduli and the associated CP-violating phases

$$
\tilde{\lambda}_{i j}=\left|\tilde{\lambda}_{i j}\right| e^{i \zeta_{i j}}
$$

We now turn to the determination of the expressions for the effective neutrino magnetic moment associated to the experimental setup of interest. We start with the case of solar neutrino experiments, generalizing previous results [40,41] to a general expression in the mass basis

$$
\left(\mu_{\nu, \mathrm{eff}}^{M}\right)^{2}\left(L, E_{\nu}\right)=\sum_{j}\left|\sum_{i} K_{\alpha i}^{*} e^{-i \Delta m_{i j}^{2} L / 2 E_{\nu}} \tilde{\lambda}_{i j}\right|^{2},
$$

where the $3 \times(3+\mathrm{m})$ rectangular matrix $K$ is the upper truncation of the $(3+\mathrm{m}) \times(3+\mathrm{m})$ unitary matrix diagonalizing the neutrinos, where $m$ is the number of sterile neutrinos [11]. We also assume that the charged leptons are in their mass-diagonal basis. The indices $i$ and $j$ run over the total number of neutrino mass eigenstates. The large baseline-distance for the case of solar neutrinos makes the interference terms vanish. Therefore, we can generalize the three-neutrino expression in ref. [42] to our case of interest, where we take just four neutrino species $(\mathrm{m}=1)$,

$$
\begin{aligned}
\left(\mu_{\nu, \mathrm{sol}}^{M}\right)^{2}= & P_{e 1}\left(\left|\tilde{\lambda}_{12}\right|^{2}+\left|\tilde{\lambda}_{13}\right|^{2}+\left|\tilde{\lambda}_{14}\right|^{2}\right)+P_{e 2}\left(\left|\tilde{\lambda}_{12}\right|^{2}+\left|\tilde{\lambda}_{23}\right|^{2}+\left|\tilde{\lambda}_{24}\right|^{2}\right) \\
& +P_{e 3}\left(\left|\tilde{\lambda}_{13}\right|^{2}+\left|\tilde{\lambda}_{23}\right|^{2}+\left|\tilde{\lambda}_{34}\right|^{2}\right)+P_{e 4}\left(\left|\tilde{\lambda}_{14}\right|^{2}+\left|\tilde{\lambda}_{24}\right|^{2}+\left|\tilde{\lambda}_{34}\right|^{2}\right),
\end{aligned}
$$

where $P_{e i}$ corresponds to the solar neutrino transition probability from the originally created $\nu_{e}$ state to the mass eigenstate $\nu_{i}$ (see ref. [23]).

For short-baseline experiments, and assuming very small mixing between active and sterile states, one can just assume at detectors the presence of pure $\nu_{e}$ and $\nu_{\mu}$ beams. In this case, the full analytical expressions for the effective neutrino magnetic moments for $\nu_{e}$ and $\nu_{\mu}$ in the mass basis are lengthy. For the reader's convenience, we give them in the form

$$
\begin{aligned}
& \left(\mu_{\nu_{e}}^{M}\right)^{2}=\sum_{\kappa} \tilde{\Lambda}_{\kappa}^{2} C_{\nu_{e}}^{\kappa}\left(\theta_{i j}, \zeta_{i j}, \delta_{i j}\right), \\
& \left(\mu_{\nu_{\mu}}^{M}\right)^{2}=\sum_{\kappa} \tilde{\Lambda}_{\kappa}^{2} C_{\nu_{\mu}}^{\kappa}\left(\theta_{i j}, \zeta_{i j}, \delta_{i j}\right),
\end{aligned}
$$

where the index $\kappa$ runs over the 21 components listed in table 1 (for $\alpha=e$ ) and table 2 (for $\alpha=\mu$ ). Here, $\tilde{\Lambda}_{k}^{2}$ has dimensions of $\mu_{B}^{2}$ and represents the product of two TMMs (all possible combinations of $\left.\left|\tilde{\lambda}_{i j}\right|\left|\tilde{\lambda}_{i^{\prime} j^{\prime}}\right|\right)$, and $C_{\nu_{\alpha}}^{\kappa}\left(\theta_{i j}, \zeta_{i j}, \delta_{i j}\right)$ denotes the associated coefficients. At this point, we should stress that the expressions in eq. (2.6) remain the same for the case of antineutrinos. Notice that, when the sterile neutrino parameters are neglected, eq. (2.6)

\footnotetext{
${ }^{1}$ As an academic note, we mention that in the (now unphysical) limit where the "active" and "sterile" masses coincide, this interaction would correspond to the often discussed "Dirac" neutrino magnetic moment.
} 


\begin{tabular}{|c|c|}
\hline$\tilde{\Lambda}_{\kappa}^{2}$ & $C_{\nu_{e}}^{\kappa}\left(\theta_{i j}, \zeta_{i j}, \delta_{i j}\right)$ \\
\hline$\left|\tilde{\lambda}_{12}\right|^{2}$ & $c_{13}^{2} c_{14}^{2}$ \\
$\left|\tilde{\lambda}_{13}\right|^{2}$ & $c_{14}^{2}\left(c_{12}^{2} c_{13}^{2}+s_{13}^{2}\right)$ \\
$\left|\tilde{\lambda}_{14}\right|^{2}$ & $c_{12}^{2} c_{13}^{2} c_{14}^{2}+s_{14}^{2}$ \\
$\left|\tilde{\lambda}_{23}\right|^{2}$ & $c_{14}^{2}\left(s_{12}^{2} c_{13}^{2}+s_{13}^{2}\right)$ \\
$\left|\tilde{\lambda}_{24}\right|^{2}$ & $s_{12}^{2} c_{13}^{2} c_{14}^{2}+s_{14}^{2}$ \\
$\left|\tilde{\lambda}_{34}\right|^{2}$ & $c_{14}^{2} s_{13}^{2}+s_{14}^{2}$ \\
$\left|\tilde{\lambda}_{12}\right|\left|\tilde{\lambda}_{13}\right|$ & $-c_{14}^{2} s_{12} \sin 2 \theta_{13} \cos \left(\delta-\zeta_{12}+\zeta_{13}\right)$ \\
$\left|\tilde{\lambda}_{12}\right|\left|\tilde{\lambda}_{14}\right|$ & $c_{13} s_{12} \sin 2 \theta_{14} \cos \left(\delta_{14}-\zeta_{12}+\zeta_{14}\right)$ \\
$\left|\tilde{\lambda}_{12}\right|\left|\tilde{\lambda}_{23}\right|$ & $-c_{14}^{2} c_{12} \sin 2 \theta_{13} \cos \left(\delta-\zeta_{12}+\zeta_{23}\right)$ \\
$\left|\tilde{\lambda}_{12}\right|\left|\tilde{\lambda}_{24}\right|$ & $-c_{13} c_{12} \sin 2 \theta_{14} \cos \left(\delta_{14}-\zeta_{12}+\zeta_{24}\right)$ \\
$\left|\tilde{\lambda}_{12}\right|\left|\tilde{\lambda}_{34}\right|$ & 0 \\
$\left|\tilde{\lambda}_{13}\right|\left|\tilde{\lambda}_{14}\right|$ & $-s_{13} \sin 2 \theta_{14} \cos \left(\delta-\delta_{14}+\zeta_{13}-\zeta_{14}\right)$ \\
$\left|\tilde{\lambda}_{13}\right|\left|\tilde{\lambda}_{23}\right|$ & $-c_{13}^{2} c_{14}^{2} \sin 2 \theta_{12} \cos \left(\zeta_{13}-\zeta_{23}\right)$ \\
$\left|\tilde{\lambda}_{13}\right|\left|\tilde{\lambda}_{24}\right|$ & 0 \\
$\left|\tilde{\lambda}_{13}\right|\left|\tilde{\lambda}_{34}\right|$ & $c_{12} c_{13} \sin 2 \theta_{14} \cos \left(\delta_{14}-\zeta_{13}+\zeta_{34}\right)$ \\
$\left|\tilde{\lambda}_{14}\right|\left|\tilde{\lambda}_{23}\right|$ & 0 \\
$\left|\tilde{\lambda}_{14}\right|\left|\tilde{\lambda}_{24}\right|$ & $c_{13}^{2} c_{14}^{2} \sin 2 \theta_{12} \cos \left(\zeta_{14}-\zeta_{24}\right)$ \\
$\left|\tilde{\lambda}_{14}\right|\left|\tilde{\lambda}_{34}\right|$ & $c_{12} c_{14}^{2} \sin 2 \theta_{13} \cos \left(\delta-\zeta_{14}+\zeta_{34}\right)$ \\
$\left|\tilde{\lambda}_{23}\right|\left|\tilde{\lambda}_{24}\right|$ & $s_{13} \sin 2 \theta_{14} \cos \left(\delta-\delta_{14}+\zeta_{23}-\zeta_{24}\right)$ \\
$\left|\tilde{\lambda}_{23}\right|\left|\tilde{\lambda}_{34}\right|$ & $-c_{13} s_{12} \sin 2 \theta_{14} \cos \left(\delta_{14}-\zeta_{23}+\zeta_{34}\right)$ \\
$\left|\tilde{\lambda}_{24}\right|\left|\tilde{\lambda}_{34}\right|$ & $c_{14}^{2} s_{12} \sin 2 \theta_{13} \cos \left(\delta-\zeta_{24}+\zeta_{34}\right)$ \\
\hline
\end{tabular}

Table 1. TMMs and the corresponding coefficients entering in the expression of the effective magnetic moment in eq. (2.6) for electron neutrinos.

reduces to the three-neutrino mixing expression obtained in ref. [43]. Since we are interested in TMMs from active to sterile states, in the following calculations we will concentrate only on the relevant terms containing $\tilde{\lambda}_{i 4}$. Thus, ignoring the active-active terms with $\tilde{\lambda}_{12}, \tilde{\lambda}_{13}$ and $\tilde{\lambda}_{23}$ as well as the cross terms $\tilde{\lambda}_{i 4} \tilde{\lambda}_{j 4}$, the effective magnetic moment responsible for $\nu_{e} \rightarrow \nu_{s}$ and $\bar{\nu}_{e} \rightarrow \nu_{s}$ transitions reduces to

$$
\left(\mu_{\nu_{e} \rightarrow \nu_{s}}^{M}\right)^{2}=\left|\tilde{\lambda}_{14}\right|^{2}\left(c_{12}^{2} c_{13}^{2} c_{14}^{2}+s_{14}^{2}\right)+\left|\tilde{\lambda}_{24}\right|^{2}\left(s_{12}^{2} c_{13}^{2} c_{14}^{2}+s_{14}^{2}\right)+\left|\tilde{\lambda}_{34}\right|^{2}\left(s_{13}^{2} c_{14}^{2}+s_{14}^{2}\right) .
$$

Here, $c_{i j}=\cos \theta_{i j}$ and $s_{i j}=\sin \theta_{i j}$, with $\theta_{i j}$ being the different neutrino mixing angles and 


\begin{tabular}{|c|c|}
\hline$\tilde{\Lambda}^{2}$ & $C_{\nu_{\mu}}\left(\theta_{i j}, \zeta_{i j}, \delta_{i j}\right)$ \\
\hline$\left|\tilde{\lambda}_{12}\right|^{2}$ & $c_{24} s_{14} s_{23} s_{24} \sin 2 \theta_{13} \cos \left(\delta-\delta_{14}\right)+c_{24}^{2}\left(c_{23}^{2}+s_{13}^{2} s_{23}^{2}\right)+c_{13}^{2} s_{14}^{2} s_{24}^{2}$ \\
\hline$\left|\tilde{\lambda}_{13}\right|^{2}$ & $\begin{aligned} & c_{24}^{2}\left[c_{23} s_{13} s_{23} \sin 2 \theta_{12} \cos \delta+c_{23}^{2} s_{12}^{2}+s_{23}^{2}\left(c_{12}^{2} s_{13}^{2}+c_{13}^{2}\right)\right] \\
+ & c_{13} s_{14} \sin 2 \theta_{24}\left[c_{12} c_{23} s_{12} \cos \delta_{14}+\left(c_{12}^{2}-1\right) s_{13} s_{23} \cos \left(\delta-\delta_{14}\right)\right]+s_{14}^{2} s_{24}^{2}\left(c_{12}^{2} c_{13}^{2}+s_{13}^{2}\right)\end{aligned}$ \\
\hline$\left|\tilde{\lambda}_{14}\right|^{2}$ & $\begin{array}{l}c_{24}^{2}\left[c_{23} s_{13} s_{23} \sin 2 \theta_{12} \cos \delta+c_{23}^{2} s_{12}^{2}+c_{12}^{2} s_{13}^{2} s_{23}^{2}\right] \\
+c_{12} c_{13} s_{14} \sin 2 \theta_{24}\left[c_{23} s_{12} \cos \delta_{14}+c_{12} s_{13} s_{23} \cos \left(\delta-\delta_{14}\right)\right]+s_{24}^{2}\left(c_{12}^{2} c_{13}^{2} s_{14}^{2}+c_{14}^{2}\right)\end{array}$ \\
\hline$\left|\tilde{\lambda}_{23}\right|^{2}$ & $\begin{array}{l}c_{24}^{2}\left[-c_{23} s_{13} s_{23} \sin 2 \theta_{12} \cos \delta+s_{23}^{2}\left(c_{13}^{2}+s_{12}^{2} s_{13}^{2}\right)+c_{12}^{2} c_{23}^{2}\right] \\
-c_{13} s_{14} \sin 2 \theta_{24}\left[c_{12} c_{23} s_{12} \cos \delta_{14}-\left(s_{12}^{2}-1\right) s_{13} s_{23} \cos \left(\delta-\delta_{14}\right)\right]+s_{14}^{2} s_{24}^{2}\left(c_{13}^{2} s_{12}^{2}+s_{13}^{2}\right)\end{array}$ \\
\hline$\left|\tilde{\lambda}_{24}\right|^{2}$ & $\begin{array}{l}c_{24}^{2}\left[-c_{23} s_{13} s_{23} \sin 2 \theta_{12} \cos \delta+c_{12}^{2} c_{23}^{2}+s_{12}^{2} s_{13}^{2} s_{23}^{2}\right] \\
+c_{13} s_{12} s_{14} \sin 2 \theta_{24}\left[s_{12} s_{13} s_{23} \cos \left(\delta-\delta_{14}\right)-c_{12} c_{23} \cos \delta_{14}\right]+s_{24}^{2}\left(c_{13}^{2} s_{12}^{2} s_{14}^{2}+c_{14}^{2}\right)\end{array}$ \\
\hline$\left|\tilde{\lambda}_{34}\right|^{2}$ & $-c_{24} s_{14} s_{24} s_{23} \sin 2 \theta_{13} \cos \left(\delta-\delta_{14}\right)+c_{13}^{2} c_{24}^{2} s_{23}^{2}+s_{24}^{2}\left(c_{14}^{2}+s_{13}^{2} s_{14}^{2}\right)$ \\
\hline$\left|\tilde{\lambda}_{12}\right|\left|\tilde{\lambda}_{13}\right|$ & $\begin{aligned}-s_{12}[ & -c_{13}^{2} s_{14} s_{23} \sin 2 \theta_{24} \cos \left(\delta_{14}-\zeta_{12}+\zeta_{13}\right) \\
& \left.+\left(s_{14}^{2} s_{24}^{2}-c_{24}^{2} s_{23}^{2}\right) \sin 2 \theta_{13} \cos \left(\delta-\zeta_{12}+\zeta_{13}\right)+s_{13}^{2} s_{14} s_{23} \sin 2 \theta_{24} \cos \left(2 \delta-\delta_{14}-\zeta_{12}+\zeta_{13}\right)\right] \\
& -2 c_{12} c_{23} c_{24}\left[c_{13} c_{24} s_{23} \cos \left(\zeta_{12}-\zeta_{13}\right)-s_{13} s_{14} s_{24} \cos \left(\delta-\delta_{14}-\zeta_{12}+\zeta_{13}\right)\right]\end{aligned}$ \\
\hline$\left|\tilde{\lambda}_{12}\right|\left|\tilde{\lambda}_{14}\right|$ & $-2 c_{14} s_{24}\left\{s_{12}\left[c_{24} s_{13} s_{23} \cos \left(\delta-\zeta_{12}+\zeta_{14}\right)+c_{13} s_{14} s_{24} \cos \left(\delta_{14}-\zeta_{12}+\zeta_{14}\right)\right]-c_{12} c_{23} c_{24} \cos \left(\zeta_{12}-\zeta_{14}\right)\right\}$ \\
\hline$\left|\tilde{\lambda}_{12}\right|\left|\tilde{\lambda}_{23}\right|$ & $\begin{array}{l}c_{12} c_{13}^{2} s_{14} s_{23} \sin 2 \theta_{24} \cos \left(\delta_{14}-\zeta_{12}+\zeta_{23}\right) \\
+c_{13}\left[2 c_{12} s_{13}\left(c_{24}^{2} s_{23}^{2}-s_{14}^{2} s_{24}^{2}\right) \cos \left(\delta-\zeta_{12}+\zeta_{23}\right)+c_{24}^{2} s_{12} \cos \left(\zeta_{12}-\zeta_{23}\right) \sin 2 \theta_{23}\right] \\
-s_{13} s_{14} \sin 2 \theta_{24}\left[c_{23} s_{12} \cos \left(\delta-\delta_{14}-\zeta_{12}+\zeta_{23}\right)+c_{12} s_{13} s_{23} \cos \left(2 \delta-\delta_{14}-\zeta_{12}+\zeta_{23}\right)\right]\end{array}$ \\
\hline$\left|\tilde{\lambda}_{12}\right|\left|\tilde{\lambda}_{24}\right|$ & $2 c_{14} s_{24}\left\{c_{12}\left[c_{24} s_{13} s_{23} \cos \left(\delta-\zeta_{12}+\zeta_{24}\right)+c_{13} s_{14} s_{24} \cos \left(\delta_{14}-\zeta_{12}+\zeta_{24}\right)\right]+c_{23} c_{24} s_{12} \cos \left(\zeta_{12}-\zeta_{24}\right)\right\}$ \\
\hline$\left|\tilde{\lambda}_{12}\right|\left|\tilde{\lambda}_{34}\right|$ & 0 \\
\hline$\left|\tilde{\lambda}_{13}\right|\left|\tilde{\lambda}_{14}\right|$ & $2 c_{14} s_{24}\left[s_{13} s_{14} s_{24} \cos \left(\delta-\delta_{14}+\zeta_{13}-\zeta_{14}\right)-c_{13} c_{24} s_{23} \cos \left(\zeta_{13}-\zeta_{14}\right)\right]$ \\
\hline$\left|\tilde{\lambda}_{13}\right|\left|\tilde{\lambda}_{23}\right|$ & $\begin{array}{l}{\left[-2 c_{13} c_{24} s_{13} s_{14} s_{23} s_{24} \cos \left(\delta-\delta_{14}\right)+c_{24}^{2}\left(c_{23}^{2}-s_{13}^{2} s_{23}^{2}\right)-c_{13}^{2} s_{14}^{2} s_{24}^{2}\right] \sin 2 \theta_{12} \cos \left(\zeta_{13}-\zeta_{23}\right)} \\
+2 c_{23} c_{24} c_{12}^{2}\left[c_{24} s_{13} s_{23} \cos \left(\delta-\zeta_{13}+\zeta_{23}\right)+c_{13} s_{14} s_{24} \cos \left(\delta_{14}-\zeta_{13}+\zeta_{23}\right)\right] \\
-2 c_{23} c_{24} s_{12}^{2}\left[c_{24} s_{13} s_{23} \cos \left(\delta+\zeta_{13}-\zeta_{23}\right)+c_{13} s_{14} s_{24} \cos \left(\delta_{14}+\zeta_{13}-\zeta_{23}\right)\right]\end{array}$ \\
\hline$\left|\tilde{\lambda}_{13}\right|\left|\tilde{\lambda}_{24}\right|$ & 0 \\
\hline$\left|\tilde{\lambda}_{13}\right|\left|\tilde{\lambda}_{34}\right|$ & $-2 c_{14} s_{24}\left\{c_{12}\left[c_{24} s_{13} s_{23} \cos \left(\delta-\zeta_{13}+\zeta_{34}\right)+c_{13} s_{14} s_{24} \cos \left(\delta_{14}-\zeta_{13}+\zeta_{34}\right)\right]+c_{23} c_{24} s_{12} \cos \left(\zeta_{13}-\zeta_{34}\right)\right\}$ \\
\hline$\left|\tilde{\lambda}_{14}\right|\left|\tilde{\lambda}_{23}\right|$ & 0 \\
\hline$\left|\tilde{\lambda}_{14}\right|\left|\tilde{\lambda}_{24}\right|$ & $\begin{array}{l}{\left[2 c_{13} c_{24} s_{13} s_{14} s_{23} s_{24} \cos \left(\delta-\delta_{14}\right)+c_{24}^{2}\left(s_{13}^{2} s_{23}^{2}-c_{23}^{2}\right)+c_{13}^{2} s_{14}^{2} s_{24}^{2}\right] \sin 2 \theta_{12} \cos \left(\zeta_{14}-\zeta_{24}\right)} \\
-2 c_{23} c_{24} c_{12}^{2}\left[c_{24} s_{13} s_{23} \cos \left(\delta-\zeta_{14}+\zeta_{24}\right)+c_{13} s_{14} s_{24} \cos \left(\delta_{14}-\zeta_{14}+\zeta_{24}\right)\right] \\
+2 c_{23} c_{24} s_{12}^{2}\left[c_{24} s_{13} s_{23} \cos \left(\delta+\zeta_{14}-\zeta_{24}\right)+c_{13} s_{14} s_{24} \cos \left(\delta_{14}+\zeta_{14}-\zeta_{24}\right)\right]\end{array}$ \\
\hline$\left|\tilde{\lambda}_{14}\right|\left|\tilde{\lambda}_{34}\right|$ & $\begin{array}{l}\quad-c_{12} c_{13}^{2} s_{14} s_{23} \sin 2 \theta_{24} \cos \left(\delta_{14}-\zeta_{14}+\zeta_{34}\right) \\
-c_{13}\left[2 c_{12} s_{13}\left(c_{24}^{2} s_{23}^{2}-s_{14}^{2} s_{24}^{2}\right) \cos \left(\delta-\zeta_{14}+\zeta_{34}\right)+c_{24}^{2} s_{12} \cos \left(\zeta_{14}-\zeta_{34}\right) \sin 2 \theta_{23}\right] \\
+s_{13} s_{14} \sin 2 \theta_{24}\left[c_{23} s_{12} \cos \left(\delta-\delta_{14}-\zeta_{14}+\zeta_{34}\right)+c_{12} s_{13} s_{23} \cos \left(2 \delta-\delta_{14}-\zeta_{14}+\zeta_{34}\right)\right]\end{array}$ \\
\hline$\left|\tilde{\lambda}_{23}\right|\left|\tilde{\lambda}_{24}\right|$ & $2 c_{14} s_{24}\left[c_{13} c_{24} s_{23} \cos \left(\zeta_{23}-\zeta_{24}\right)-s_{13} s_{14} s_{24} \cos \left(\delta-\delta_{14}+\zeta_{23}-\zeta_{24}\right)\right]$ \\
\hline$\left|\tilde{\lambda}_{23}\right|\left|\tilde{\lambda}_{34}\right|$ & $2 c_{14} s_{24}\left\{s_{12}\left[c_{24} s_{13} s_{23} \cos \left(\delta-\zeta_{23}+\zeta_{34}\right)+c_{13} s_{14} s_{24} \cos \left(\delta_{14}-\zeta_{23}+\zeta_{34}\right)\right]-c_{12} c_{23} c_{24} \cos \left(\zeta_{23}-\zeta_{34}\right)\right\}$ \\
\hline$\left|\tilde{\lambda}_{24}\right|\left|\tilde{\lambda}_{34}\right|$ & $\begin{aligned} s_{12}[ & -c_{13}^{2} s_{14} s_{23} \sin 2 \theta_{24} \cos \left(\delta_{14}-\zeta_{24}+\zeta_{34}\right)+\left(s_{14}^{2} s_{24}^{2}-c_{24}^{2} s_{23}^{2}\right) \sin 2 \theta_{13} \cos \left(\delta-\zeta_{24}+\zeta_{34}\right) \\
& \left.+s_{13}^{2} s_{14} s_{23} \sin 2 \theta_{24} \cos \left(2 \delta-\delta_{14}-\zeta_{24}+\zeta_{34}\right)\right] \\
& +2 c_{12} c_{23} c_{24}\left[c_{13} c_{24} s_{23} \cos \left(\zeta_{24}-\zeta_{34}\right)-s_{13} s_{14} s_{24} \cos \left(\delta-\delta_{14}-\zeta_{24}+\zeta_{34}\right)\right]\end{aligned}$ \\
\hline
\end{tabular}

Table 2. TMMs and corresponding coefficients for the calculation of eq. (2.6) for muon neutrinos. 
$\delta_{i j}$ denoting the corresponding $\mathrm{CP}$ violating phases present in the four-neutrino mixing matrix. Similarly, for the case of $\nu_{\mu} \rightarrow \nu_{s}$ or $\bar{\nu}_{\mu} \rightarrow \nu_{s}$ transitions, we get

$$
\begin{aligned}
& \left(\mu_{\nu_{\mu} \rightarrow \nu_{s}}^{M}\right)^{2}=\left|\tilde{\lambda}_{14}\right|^{2}\left[c_{24}^{2}\left(\sin 2 \theta_{12} s_{13} c_{23} s_{23} \cos \delta+s_{12}^{2} c_{23}^{2}+c_{12}^{2} s_{13}^{2} s_{23}^{2}\right)\right. \\
& \left.+c_{12} c_{13} s_{14} \sin 2 \theta_{24}\left[s_{12} c_{23} \cos \delta_{14}+c_{12} s_{13} s_{23} \cos \left(\delta-\delta_{14}\right)\right]+s_{24}^{2}\left(c_{12}^{2} c_{13}^{2} s_{14}^{2}+c_{14}^{2}\right)\right] \\
& +\left|\tilde{\lambda}_{24}\right|^{2}\left[c_{24}^{2}\left(-\sin 2 \theta_{12} s_{13} c_{23} s_{23} \cos \delta+c_{12}^{2} c_{23}^{2}+s_{12}^{2} s_{13}^{2} s_{23}^{2}\right)\right. \\
& \left.+s_{12} c_{13} s_{14} \sin 2 \theta_{24}\left[s_{12} s_{13} s_{23} \cos \left(\delta-\delta_{14}\right)-c_{12} c_{23} \cos \delta_{14}\right]+s_{24}^{2}\left(s_{12}^{2} c_{13}^{2} s_{14}^{2}+c_{14}^{2}\right)\right] \\
& +\left|\tilde{\lambda}_{34}\right|^{2}\left[-\sin 2 \theta_{13} s_{23} s_{14} c_{24} s_{24} \cos \left(\delta-\delta_{14}\right)+c_{13}^{2} s_{23}^{2} c_{24}^{2}+s_{24}^{2}\left(s_{13}^{2} s_{14}^{2}+c_{14}^{2}\right)\right] .
\end{aligned}
$$

Assuming a small active-sterile neutrino mixing, $\sin ^{2} \theta_{i 4} \leq 0.01$, the last two expressions simplify considerably and read

$$
\begin{aligned}
\left(\mu_{\nu_{e} \rightarrow \nu_{s}}^{M}\right)^{2} \approx & \left|\tilde{\lambda}_{14}\right|^{2} c_{13}^{2} c_{12}^{2}+\left|\tilde{\lambda}_{24}\right|^{2} c_{13}^{2} s_{12}^{2}+\left|\tilde{\lambda}_{34}\right|^{2} s_{13}^{2} \\
\left(\mu_{\nu_{\mu} \rightarrow \nu_{s}}^{M}\right)^{2} \approx & \left|\tilde{\lambda}_{14}\right|^{2}\left(c_{23} s_{13} s_{23} \sin 2 \theta_{12} \cos \delta+c_{23}^{2} s_{12}^{2}+c_{12}^{2} s_{13}^{2} s_{23}^{2}\right) \\
& +\left|\tilde{\lambda}_{24}\right|^{2}\left(-c_{23} s_{13} s_{23} \sin 2 \theta_{12} \cos \delta+c_{23}^{2} c_{12}^{2}+s_{12}^{2} s_{13}^{2} s_{23}^{2}\right)+\left|\tilde{\lambda}_{34}\right|^{2} c_{13}^{2} s_{23}^{2} .
\end{aligned}
$$

In what follows, for our analysis involving reactor neutrino experiments $\left(\bar{\nu}_{e}\right.$ source) or ${ }^{51} \mathrm{Cr}$ experiments $\left(\nu_{e}\right.$ source), the effective neutrino magnetic moment will be given by eq. (2.9). Similarly, for the analysis of the COHERENT experiment involving piondecay-at-rest neutrinos, eq. (2.9) is relevant for the $\nu_{e}$ component of the SNS beam and eq. (2.10) for the $\nu_{\mu}$ or $\bar{\nu}_{\mu}$ components. Before closing this discussion, we comment on the phase counting. As shown in ref. [44], for the case of $n$ Majorana neutrinos the number of physical phases is $n(n-2)$, hence we have in general 8 physical phases expected in the present study (see tables 1 and 2).

\section{2 $\mathrm{CE} \nu \mathrm{NS}$ and neutrino-electron scattering in the SM}

Assuming only SM interactions, the differential CE $\nu$ NS cross-section for the nuclear recoil energy $E_{r}$, is written as [45]

$$
\left(\frac{\mathrm{d} \sigma}{\mathrm{d} E_{r}}\right)_{\mathrm{SM}}=\frac{G_{F}^{2} M}{\pi}\left(\mathcal{Q}_{V}\right)^{2}\left[1-\frac{E_{r}}{E_{\nu}}-\frac{M E_{r}}{2 E_{\nu}^{2}}\right]
$$

where $G_{F}, E_{\nu}$, and $M$ stand for the Fermi constant, the incident neutrino energy, and the nuclear mass, respectively. Here $\mathcal{Q}_{W}^{V}$ denotes the weak charge, defined as [46]

$$
\mathcal{Q}_{V}=\left[\left(\frac{1}{2}-2 \sin ^{2} \theta_{W}\right) Z F_{p}\left(Q^{2}\right)-\frac{1}{2} N F_{n}\left(Q^{2}\right)\right],
$$

with the weak mixing angle being $\sin ^{2} \theta_{W}=0.2312$. It is noteworthy that the main uncertainty in the theoretical $\mathrm{CE} \nu \mathrm{NS}$ calculation arises from nuclear physics effects, which may limit the experimental sensitivity for searches of physics beyond the SM (for a detailed 
analysis, see ref. [47]). In the latter expression, nuclear structure corrections are taken into account through the nuclear form factors for protons $F_{p}\left(Q^{2}\right)$ and neutrons $F_{n}\left(Q^{2}\right)$. The magnitude of the momentum transfer is $Q=\sqrt{2 M E_{r}}$. We employ the Helm parametrization $^{2}$

$$
F_{p, n}\left(Q^{2}\right)=3 \frac{j_{1}\left(Q R_{0}\right)}{Q R_{0}} \exp \left(-Q^{2} s^{2} / 2\right)
$$

where $j_{1}(x)=\sin (x) / x^{2}-\cos (x) / x$ is the spherical Bessel function of order one, and $R_{0}^{2}=\frac{5}{3}\left(R_{p, n}^{2}-3 s^{2}\right)$ with $R_{p, n}$ the proton and neutron r.m.s. radii and $s=0.9 \mathrm{fm}$ the surface thickness.

Turning to elastic neutrino electron scattering, E $\nu \mathrm{ES}$, for a given neutrino flavor $\alpha=$ $e, \mu, \tau$, the corresponding SM differential cross-section as a function of the electron recoil energy is given as

$$
\left(\frac{\mathrm{d} \sigma_{\nu_{\alpha}-e^{-}}}{\mathrm{d} E_{r}}\right)_{\mathrm{SM}}^{\text {free }}=\frac{2 G_{F}^{2} m_{e}}{\pi}\left[g_{L}^{2}+g_{R}^{2}\left(1-\frac{E_{r}}{E_{\nu}}\right)^{2}-g_{L} g_{R} \frac{m_{e} E_{r}}{E_{\nu}^{2}}\right]
$$

where we assume the free-electron approximation and $m_{e}$ is the electron mass. Here, the left-handed, $g_{L}=\left(g_{V}+g_{A}\right) / 2$, and right-handed couplings, $g_{R}=\left(g_{V}-g_{A}\right) / 2$, are expressed in terms of the vector and axial vector couplings with

$$
\begin{aligned}
& g_{V}=-1 / 2+2 \sin ^{2} \theta_{W}+\delta_{\alpha e}, \\
& g_{A}=-1 / 2+\delta_{\alpha e} .
\end{aligned}
$$

The cross-section for antineutrino scattering off electrons is obtained by exchanging $g_{L} \leftrightarrow$ $g_{R}$. Notice that the factor $\delta_{\alpha e}$ in eq. (2.15) is present only when electron (anti)neutrinos are involved. In this case, the cross-section receives contributions from both neutral-current and charged-current interactions, unlike the case of $\alpha=\mu, \tau$, where the interaction is purely neutral-current.

As we mentioned, eq. (2.14) applies to E $\nu \mathrm{ES}$ with free electrons. To take into account electron binding effects in the target material of a given experiment, we weight the free E $\nu \mathrm{ES}$ cross-section by adopting the step approximation $[49]^{3}$

$$
\left(\frac{\mathrm{d} \sigma_{\nu_{\alpha}-e^{-}}}{\mathrm{d} E_{r}}\right)_{\mathrm{SM}}=\frac{1}{Z} \sum_{i=1}^{Z} \Theta\left(E_{r}-B_{i}\right)\left(\frac{\mathrm{d} \sigma_{\nu_{\alpha}-e^{-}}}{\mathrm{d} E_{r}}\right)_{\mathrm{SM}}^{\text {free }},
$$

where $B_{i}$ is the binding energy of the $i$ th atomic (sub)shell. This way, one suppresses the free $\mathrm{E} \nu \mathrm{EScross}-\mathrm{section}$ and quantifies the impact of the atomic ionization energy levels. This calculation takes into account only those electrons that can be ionized by an energy deposition $E_{r}$, the modifications become important below a few $\mathrm{keV}$ recoil energies.

\footnotetext{
${ }^{2}$ Following ref. [35], for the COHERENT-CsI detector we adopt the Klein-Nystrand (KN) form factor $F_{\mathrm{KN}}=3 \frac{j_{1}\left(Q R_{A}\right)}{Q R_{A}}\left[1+\left(Q a_{k}\right)^{2}\right]^{-1}$, where $R_{A}=1.23 \times A^{1 / 3}$ and $a_{k}=0.7 \mathrm{fm}$ [48].

${ }^{3}$ Note that we normalize the suppression factor to unity.
} 


\subsection{Sterile neutrino dipole portal}

Recently, there has been some interest in the transition of an active neutrino to a massive sterile state, induced by a magnetic coupling. Assuming a spin $1 / 2$ nucleus, the corresponding $\mathrm{CE} \nu \mathrm{NS}$ cross-section reads ${ }^{4}[33]$

$$
\frac{\mathrm{d} \sigma_{\nu \mathcal{N} \rightarrow \nu_{s} \mathcal{N}}}{\mathrm{d} E_{r}}=\alpha_{\mathrm{em}} \mu_{\nu, \mathrm{eff}}^{2} Z^{2}\left[\frac{1}{E_{r}}-\frac{1}{E_{\nu}}-\frac{m_{4}^{2}}{2 E_{\nu} E_{r} M}\left(1-\frac{E_{r}}{2 E_{\nu}}+\frac{M}{2 E_{\nu}}\right)+\frac{m_{4}^{4}\left(E_{r}-M\right)}{8 E_{\nu}^{2} E_{r}^{2} M^{2}}\right] F_{p}^{2}\left(Q^{2}\right),
$$

where $\alpha_{\mathrm{em}}$ is the fine structure constant and $m_{4}$ is the sterile neutrino mass. For a spinless nucleus the differential cross-section remains essentially unchanged, i.e.

$$
\frac{\mathrm{d} \sigma_{(\operatorname{spin}=1 / 2)}}{\mathrm{d} E_{r}}-\frac{\mathrm{d} \sigma_{(\operatorname{spin}=0)}}{\mathrm{d} E_{r}}=\frac{m_{4}^{2}}{8 M E_{\nu}^{2}}\left(1+\frac{m_{4}^{2}}{M E_{r}}\right)-\frac{E_{r}}{4 E_{\nu}^{2}} .
$$

For the case of the free-electron $\mathrm{E} \nu \mathrm{ES}$ via the neutrino dipole portal, the corresponding cross-section is trivially obtained from eq. (2.17) with the substitutions $M \rightarrow m_{e}$ and $Z^{2} F_{p}^{2}\left(Q^{2}\right) \rightarrow 1$, as $[25,26]$

$$
\left(\frac{\mathrm{d} \sigma_{\nu e^{-} \rightarrow \nu_{s} e^{-}}}{\mathrm{d} E_{r}}\right)^{\text {free }}=\alpha_{\mathrm{em}} \mu_{\nu, \mathrm{eff}}^{2}\left[\frac{1}{E_{r}}-\frac{1}{E_{\nu}}-\frac{m_{4}^{2}}{2 E_{\nu} E_{r} m_{e}}\left(1-\frac{E_{r}}{2 E_{\nu}}+\frac{m_{e}}{2 E_{\nu}}\right)+\frac{m_{4}^{4}\left(E_{r}-m_{e}\right)}{8 E_{\nu}^{2} E_{r}^{2} m_{e}^{2}}\right] .
$$

As in the SM case, the E $\nu \mathrm{ES}$ cross-section is weighted with the step function in eq. (2.16). Note that for both, $\mathrm{CE} \nu \mathrm{NS}$ and $\mathrm{E} \nu \mathrm{ES}$, we recover the usual expressions for the conventional neutrino magnetic moment cross-section as $m_{4}$ approaches zero [40].

Moreover, for the case of massive final state neutrinos, one might consider the interference term between magnetic and weak interactions. Neglecting the incident neutrino mass, the corresponding cross-section for $\bar{\nu}_{e}-e^{-}$scattering can been written as [50]

$$
\left(\frac{\mathrm{d} \sigma_{\bar{\nu}_{e} e^{-} \rightarrow \nu_{s} e^{-}}}{\mathrm{d} E_{r}}\right)^{\text {interf }}=\frac{\alpha_{\mathrm{em}} G_{F} m_{4}}{\sqrt{2} E_{\nu} m_{e}} \operatorname{Re}\left[\sum_{j, n} e^{-i \frac{\Delta m_{j n}^{2} L}{2 E_{\nu}}} U_{e j} U_{e n}^{*} \tilde{\lambda}_{j 4}\left(\frac{m_{e}}{E_{\nu}}-\frac{E_{r}}{E_{\nu}}\right) Z_{n 4}^{V *}+\left(2-\frac{E_{r}}{E_{\nu}}\right) Z_{n 4}^{A *}\right],
$$

where $Z_{j k}^{V, A}=U_{e j} U_{e k}^{*}+\delta_{j k} \tilde{g}_{V, A}$ with $\tilde{g}_{V}=-1 / 2+2 \sin ^{2} \theta_{W}$ and $\tilde{g}_{A}=-1 / 2 .{ }^{5}$ From the latter expression it can be deduced that the cross-section is proportional to $\frac{m_{4}}{m_{e}} \sin 2 \theta_{14}$ for incident $\nu_{e}$ or $\bar{\nu}_{e}$. Similarly, for incident $\nu_{\mu}$ or $\bar{\nu}_{\mu}$, the cross-section is proportional to $\frac{m_{4}}{m_{e}} c_{14} s_{24}^{2}$. For the case of CE $\nu \mathrm{NS}$, the interference cross-section can be obtained via the substitutions: $\tilde{\lambda}_{i j} \rightarrow \tilde{\lambda}_{i j} Z F_{p}\left(q^{2}\right), m_{e} \rightarrow M, \tilde{g}_{V} \rightarrow \mathcal{Q}_{V}$ and $\tilde{g}_{A} \rightarrow \mathcal{Q}_{A}{ }^{6}$

A few comments regarding the interference cross-section are in order. First, for sterile mixings of the order of $\sin ^{2} \theta_{i 4} \simeq 10^{-1}$, the interference effect will be tiny. Moreover, due

\footnotetext{
${ }^{4}$ Subdominant contributions due to a nuclear magnetic moment are neglected.

${ }^{5}$ For $\nu_{e}-e^{-}$scattering, the replacement $\tilde{g}_{A} \rightarrow-\tilde{g}_{A}$ and the appropriate complex conjugation of the mixing matrix elements should be made.

${ }^{6}$ The axial weak charge $\mathcal{Q}_{A}$ vanishes for spin-zero nuclei and of the order of $\sim 1 / A$ for nuclei with non-zero spin.
} 
to its $1 / E_{\nu}$ dependence, the cross-section is suppressed for neutrino energies above a few $\mathrm{MeV}$, since from the kinematics one always has $E_{\nu}$ larger than $m_{4}$. Hence, experiments exposed to very low-energy neutrinos as the ${ }^{51} \mathrm{Cr}$ source experiment we will discuss later, will be more sensitive to the interference effects than a reactor antineutrino experiment or a $\pi$-DAR based experiment, that will have negligible sensitivity. We finally note that the contribution from the $\mathrm{CE} \nu \mathrm{NS}$ cross-section to this process can be safely ignored, as it is suppressed by the nuclear mass. As an example, for the COHERENT experiment and $m_{4}=50 \mathrm{MeV}\left(m_{4}=50 \mathrm{keV}\right)$, the interference-induced event rates are suppressed by seven (five) orders of magnitude compared to the purely magnetic-induced events.

\subsection{Event rates}

In what follows, we simulate the expected signal for various $\mathrm{CE} \nu \mathrm{NS}$ and $\mathrm{E} \nu \mathrm{ES}$ experimental probes. The differential event rate is written as

$$
\frac{\mathrm{d} N}{\mathrm{~d} E_{r}}=N_{T} \times \mathcal{E} \times \mathcal{A}\left(E_{r}\right) \int_{E_{\nu}^{\min }}^{E_{\nu}^{\max }} \frac{\mathrm{d} \sigma}{\mathrm{d} E_{R}}\left(E_{\nu}, E_{r}\right) \frac{\mathrm{d} \phi}{\mathrm{d} E_{\nu}}\left(E_{\nu}\right) \mathrm{d} E_{\nu},
$$

where $N_{T}$ denotes the number of targets (nuclei or electrons) per $\mathrm{kg}, \mathcal{E}$ is the exposure in units of kg.yr and $\mathcal{A}\left(E_{r}\right)$ represents the efficiency. The differential cross-section is given by eqs. (2.11) and (2.14) for SM interactions only, or eqs. (2.17) and (2.19) for the new physics scenario under consideration, while $\mathrm{d} \phi / \mathrm{d} E_{\nu}$ is the relevant neutrino flux for each experiment (see section 3). Finally, the upper integration limit is flux-dependent, while the lower integration limit is different for each process and given by

$$
\begin{array}{lr}
E_{\nu}^{\text {min }}=\frac{1}{2}\left(E_{r}+\sqrt{E_{r}^{2}+2 M E_{r}}\right) \approx \sqrt{M E_{r} / 2}, & \text { SM CE } \nu \mathrm{NS}, \\
E_{\nu}^{\min } \approx \sqrt{M E_{r} / 2}\left(1+\frac{m_{4}^{2}}{2 M E_{r}}\right), & \text { dipole portal } \mathrm{CE} \nu \mathrm{NS}, \\
E_{\nu}^{\min }=\frac{1}{2}\left(E_{r}+\sqrt{E_{r}^{2}+2 m_{e} E_{r}}\right), & \mathrm{SM} \mathrm{E} \nu \mathrm{ES}, \\
E_{\nu}^{\min }=\frac{1}{2}\left(E_{r}+\sqrt{E_{r}^{2}+2 m_{e} E_{r}}\right)\left(1+\frac{m_{4}^{2}}{2 m_{e} E_{r}}\right), & \text { dipole portal E } \nu \mathrm{ES} .
\end{array}
$$

Notice that the limits corresponding to the sterile neutrino dipole interaction reduce to the SM ones in the limit of vanishing $m_{4}$.

\section{Experimental tests of neutrino magnetic moments}

In this section, we explore the sensitivities of various present and future $\mathrm{CE} \nu \mathrm{NS}$ and $\mathrm{E} \nu \mathrm{ES}$ experiments to the sterile neutrino TMMs. We present a brief discussion on each experimental facility, describing all the necessary experimental inputs for our analysis. We must notice that, for the first three cases in this section, we describe in detail experiments that already have reported their data, while the last sub-section will be devoted to the future proposals that could show a better sensitivity to the neutrino magnetic moment. 


\subsection{XENON1T}

The low-energy electron recoil data sample recorded with the XENON1T experiment [24] has prompted a plethora of works attempting to interpret the anomaly. In particular, these data have been discussed within the framework of flavor transition magnetic moments in ref. [23]. In the present paper we consider the sterile dipole portal, as discussed in [2527]. Before addressing the various novel experimental setups of the coming subsections and their physics possibilities, we first calibrate our procedures with previous studies on XENON1T. Besides confirming these earlier results, here we include the step function correction commented in eq. (2.16) and previously ignored in the literature, since its impact is particularly relevant in the $1-7 \mathrm{keV}$ region of the XENON1T excess. Although the most relevant solar neutrino fluxes to consider are the $p p$ and the ${ }^{7} \mathrm{Be}$ neutrinos, in our calculation we include the full solar neutrino spectrum from ref. [51]. For the reconstructed energy $E$, we assume a Gaussian resolution function with $\sigma=a \sqrt{E}+b E$ and $a=0.310 \sqrt{\mathrm{keV}}$ and $b=0.0037 \mathrm{keV}[24]$. We also take into account the experimental exposure and efficiency provided by the XENON1T collaboration [24]. For the statistical analysis, we adopt the $\chi^{2}$ function [52]

$$
\chi^{2}(\mathcal{S})=\sum_{i=1}^{29}\left(\frac{N_{i}^{\text {exp }}-N_{i}^{\text {theor }}(\mathcal{S})}{\sigma_{i}}\right)^{2}+\left(\frac{\mathrm{a}_{1}-1}{0.03}\right)^{2}+\left(\frac{\mathrm{a}_{2}-1}{0.026}\right)^{2},
$$

where $N_{i}^{\text {exp }}$ is the observed number of events and $N_{i}^{\text {theor }}$ is the theoretical number of new physics events including backgrounds $B_{0}$. We include all the experimental data and errors from the data release in ref. [24]. We also allow the overall normalization of the background $\left(a_{2}\right)$ and the efficiency $\left(a_{1}\right)$ to float with a Gaussian error of $2.6 \%$ and $3 \%$, respectively.

The above XENON1T exercise has motivated us to explore the sterile neutrino dipole portal scenario within a broader context, using currently available low-energy CE $\nu \mathrm{NS}$ and $\mathrm{E} \nu \mathrm{ES}$ data, to which we now turn.

\subsection{COHERENT}

The COHERENT collaboration has reported the $\mathrm{CE} \nu \mathrm{NS}$ detection in CsI [35] and liquid argon (LAr) [36] detectors. In this case, for the incoming neutrino flux, we consider the Michel spectrum, which describes the $\nu_{e}, \nu_{\mu}$, and $\bar{\nu}_{\mu}$ energy spectra generated from pion decay at rest ( $\pi$-DAR) for the Spallation Neutron Source (SNS) [53]. The $\pi$-DAR flux is normalized to $\eta=r N_{\text {POT }} / 4 \pi L^{2}$, where $L$ is the baseline, while $N_{\text {POT }}$ and $r$ denote the number of protons on target (POT) and the number of neutrinos per flavor per POT, respectively. ${ }^{7}$ For the $14.57 \mathrm{~kg}$ CsI detector, we calculate the theoretical signal as a function of the nuclear recoil energy in $\mathrm{keV}_{\mathrm{nr}}$. To convert this signal into the electron-equivalent energy space, $\mathrm{keV}_{\text {ee }}$, we use the new energy-dependent quenching factor, recently reported in ref. [54] (for its impact on physics beyond the SM, see ref. [55]). Finally, using the light yield $\mathrm{LY}=13.348 \mathrm{PE} / \mathrm{keV}_{\mathrm{ee}}$ [56], the signal is converted into a photoelectron (PE) spectrum, which we compare to the experimental data. We proceed in an analogous way for the

\footnotetext{
${ }^{7}$ The values for COHERENT-CsI and CENNS-10 detectors are taken from refs. [35, 36].
} 
case of the $24 \mathrm{~kg}$ CENNS-10 detector subsystem of COHERENT (hereafter COHERENTLAr). We first evaluate the expected signal in $\mathrm{keV}_{\text {ee }}$ using the reported quenching factor, $\mathrm{QF}=0.246+7.8 \times 10^{-4} \mathrm{keV}_{\mathrm{nr}}^{-1} E_{r}$. Afterwards, following ref. [57], the signal is converted into the reconstructed energy, $E$, using a normalized Gaussian function with resolution power $\sigma / E=0.58 / \sqrt{E\left(\mathrm{keV}_{\mathrm{ee}}\right)}$.

For our statistical analysis, we consider the experimental $\mathrm{CE} \nu \mathrm{NS}$ data from the COHERENT-CsI and COHERENT-LAr measurements. Concerning the CsI detector, we base our statistical analysis on the $\chi^{2}$ function [35]

$$
\chi^{2}(\mathcal{S})=\sum_{i=4}^{15}\left(\frac{N_{i}^{\exp }-N_{i}^{\mathrm{CE} \nu \mathrm{NS}}(\mathcal{S})\left[1+\mathrm{a}_{1}\right]-B_{0 n}^{i}\left[1+\mathrm{a}_{2}\right]}{\sqrt{N_{i}^{\exp }+B_{i}^{0 n}+2 B_{i}^{s s}}}\right)^{2}+\left(\frac{\mathrm{a}_{1}}{\sigma_{\mathrm{a}_{1}}}\right)^{2}+\left(\frac{\mathrm{a}_{2}}{\sigma_{\mathrm{a}_{2}}}\right)^{2},
$$

with $N_{i}^{\exp }$ and $N_{i}^{\mathrm{CE} \nu \mathrm{NS}}$ being the measured and theoretical signal for the $i$ th bin, respectively. The analysis is restricted to the 12 bins from $i=4$ to $i=15$, corresponding to $6 \leq \mathrm{PE} \leq 30$. $B_{i}^{0 n}$ is the beam-on prompt neutron background, while $B_{i}^{s s}$ denotes the steady-state background events taken from the AC-ON data [56]. As explained in refs. $[35,55]$, the nuisance parameters $\mathrm{a}_{1}$ and $\mathrm{a}_{2}$ quantify the systematic uncertainties of the signal and background rate, respectively, with $\sigma_{\mathrm{a}_{1}}=12.8 \%$ and $\sigma_{\mathrm{a}_{2}}=25 \%$.

Concerning the COHERENT-LAr data, we focus on the analysis- $A$ of COHERENT [36], with our sensitivity analysis based on the $\chi^{2}$ function defined in ref. [58]

$$
\begin{aligned}
\chi^{2}(\mathcal{S})= & \sum_{i=1}^{12} \frac{\left(N_{i}^{\exp }-\eta_{\mathrm{CE} \nu \mathrm{NS}} N_{i}^{\mathrm{CE} \nu \mathrm{NS}}(\mathcal{S})-\eta_{\mathrm{PBRN}} B_{i}^{\mathrm{PBRN}}-\eta_{\mathrm{LBRN}} B_{i}^{\mathrm{LBRN}}\right)^{2}}{\left(\sigma_{i}^{\exp }\right)^{2}+\left[\sigma_{\mathrm{BRNES}}\left(B_{i}^{\mathrm{PBRN}}+B_{i}^{\mathrm{LBRN}}\right)\right]^{2}} \\
& +\left(\frac{\eta_{\mathrm{CE} \nu \mathrm{NS}}-1}{\sigma_{\mathrm{CE} \nu \mathrm{NS}}}\right)^{2}+\left(\frac{\eta_{\mathrm{PBRN}}-1}{\sigma_{\mathrm{PBRN}}}\right)^{2}+\left(\frac{\eta_{\mathrm{LBRN}}-1}{\sigma_{\mathrm{LBRN}}}\right)^{2}
\end{aligned}
$$

Here we consider 12 bins in the range $[0,120] \mathrm{keV}_{\text {ee }}$ of the reconstructed energy, with $10 \mathrm{keV}_{\text {ee }}$ size each. $N_{i}^{\exp }$ denotes the measured signal with uncertainty $\sigma_{i}^{\exp }$, BRNES corresponds to the Beam Related Neutron Energy Shape, while PBRN and LBRN stand for the Prompt and Late Beam-Related Neutron Background data with $\sigma_{\mathrm{PBRN}}=32 \%$ and $\sigma_{\text {LBRN }}=100 \%$, respectively, taken from ref. [57]. The Beam Related Neutron Energy Shape (BRNES) uncertainty $\sigma_{\text {BRNES }}(1.7 \%)$ and the systematic uncertainty of the signal rate $\sigma_{\mathrm{CE} \nu \mathrm{NS}}(13.4 \%)$ are taken from ref. [58].

\subsection{TEXONO}

The TEXONO collaboration has reported a measurement of elastic neutrino-electron scattering $(\mathrm{E} \nu \mathrm{ES})$ using a $187 \mathrm{~kg} \mathrm{CsI}(\mathrm{Tl})$ detector at the Kuo-Sheng Nuclear reactor [37]. In this case, we consider the reactor antineutrino distribution from [59], normalized to a total neutrino flux of $6.4 \times 10^{12} \mathrm{~cm}^{-2} \mathrm{~s}^{-1}$. For energies below $2 \mathrm{MeV}$ we adopt the theoretical estimations from ref. [60]. Our theoretical neutrino signal expected at the detector is expressed in units of events $/(\mathrm{kg} \cdot$ day $\cdot \mathrm{MeV})$ and compared with the experimental data. Our statistical analysis follows from the $\chi^{2}$ function

$$
\chi^{2}(\mathcal{S})=\sum_{i=1}^{10}\left(\frac{N_{i}^{\text {meas }}-N_{i}^{\text {new }}(\mathcal{S})[1+\mathrm{a}]}{\sigma_{i}^{\text {stat }}}\right)^{2}+\left(\frac{\mathrm{a}}{\sigma_{\text {sys }}}\right)^{2},
$$


with $N_{i}^{\text {meas }}$ standing for the detected events. We consider the reported 10 bins distributed over the recoil energy range $[3,8] \mathrm{MeV}$ as well as their associated statistical errors, $\sigma_{i}^{\text {stat }}$, as reported in ref. [37]. Systematic uncertainties are introduced through a penalty term with $\sigma_{\text {sys }}=20 \%$. As the first step in our procedure, we reproduce the TEXONO limit on the effective electron antineutrino reactor magnetic moment, $\mu_{\bar{\nu}_{e}} \leq 2.2 \times 10^{-10} \mu_{B}$, ensuring that our statistical analysis is well-calibrated with TEXONO collaboration.

\section{$3.4 \mathrm{CE} \nu \mathrm{NS}$ and $\mathrm{E} \nu \mathrm{ES}$ with a ${ }^{51} \mathrm{Cr}$ source}

We are now motivated to explore the prospects of probing new physics phenomena using the intense beam of a low-energy monochromatic $\nu_{e}{ }^{51} \mathrm{Cr}$ source through either $\mathrm{CE} \nu \mathrm{NS}$ or $\mathrm{E} \nu \mathrm{ES}$ processes. Indeed, the very low detection thresholds aimed at these facilities, make them ideal for neutrino magnetic moment searches. We first focus on a recent proposal for measuring $\mathrm{CE} \nu \mathrm{NS}$ using a $5 \mathrm{MCi}{ }^{51} \mathrm{Cr}$ source and various $\mathrm{kg}$-scale detectors with sub$\mathrm{keV}$ capabilities [38]. In accordance with the proposal, we consider a cylindrical $2000 \mathrm{~cm}^{3}$ detector for different choices of target material such as $\mathrm{Si}$, Ge, sapphire $\left(\mathrm{Al}_{2} \mathrm{O}_{3}\right)$ and calcium tungstate $\left(\mathrm{CaWO}_{4}\right)$. The detector will be placed $25 \mathrm{~cm}$ from the source, what would imply an average neutrino flux of $1.1 \times 10^{13} \mathrm{~cm}^{-2} \mathrm{~s}^{-1}$. We estimate a target mass of 4.66, 10.6, 7.96, and $12.12 \mathrm{~kg}$ for $\mathrm{Si}, \mathrm{Ge}, \mathrm{Al}_{2} \mathrm{O}_{3}$, and $\mathrm{CaWO}_{4}$, respectively. A fixed threshold of $E_{r}^{\text {thres }}=8 \mathrm{eV}_{\mathrm{nr}}$ is considered. In our simulation, we include separately the contributions from the four lines with neutrino energies $(427,432,747,752) \mathrm{keV}$ that come from the ${ }^{51} \mathrm{Cr}$ decay. Their relative strength is $(9,1,81,9) \%$. The exposure time is expected to be 2 half-lives i.e., 55.4 days [38].

In a previous work [61], we have examined the potential of a ${ }^{51} \mathrm{Cr}$-LXe detector in probing new neutral gauge bosons through $\mathrm{E} \nu \mathrm{ES}$ measurements. We considered three different experimental configurations described in ref. [39], i.e. (A, B, C): with initial radioactivity $R_{\mathrm{Cr} 51}^{0}=(5,5,10) \mathrm{MCi}$ and a time interval of $(100,50,50)$ days, respectively. In this work, we employ the same configuration and a cylindrical LXe detector, located at a distance of $1 \mathrm{~m}$ from the source, with a height and diameter of $1.38 \mathrm{~m}$, which corresponds to a total mass of about 6 tonnes [62]. The neutrino flux expected at a detector with this geometry is calculated as described in ref. [39].

Due to the lack of experimental data for estimating the sensitivity reach for this type of experiments, we employ a simplified statistical analysis based on the $\chi^{2}$ function

$$
\chi^{2}(\mathcal{S})=\sum_{i=1}^{n}\left(\frac{N_{i}^{\mathrm{SM}}-N_{i}^{\mathrm{new}}(\mathcal{S})[1+\mathrm{a}]}{\sigma_{i}^{\mathrm{stat}}}\right)^{2}+\left(\frac{\mathrm{a}}{\sigma_{\mathrm{sys}}}\right)^{2},
$$

with $\sigma_{i}^{\text {stat }}=\sqrt{N_{i}^{\mathrm{SM}}+N_{i}^{\mathrm{bg}}}$. Here we have assumed a fixed background as large as $20 \%$ of the $\mathrm{SM}$ rate, i.e. $N_{i}^{\mathrm{bg}}=\sigma_{\mathrm{bg}} N_{i}^{\mathrm{SM}}$ with $\sigma_{\mathrm{bg}}=20 \%$, while the systematic uncertainty is also taken to be $\sigma_{\text {sys }}=20 \%$. For the case of $\mathrm{CE} \nu \mathrm{NS}$, we consider $n=12$ bins within the range $\left[E_{r}^{\text {thres }}, E_{r}^{\max }\right]$ where $E_{r}^{\max }$ is the maximum recoil energy for each nuclear target. For $\mathrm{E} \nu \mathrm{ES}$ we take $5 \mathrm{keV}_{\text {ee }}$ wide bins in the range $[1,601] \mathrm{keV}_{\text {ee }}$ (see ref. [39]). 

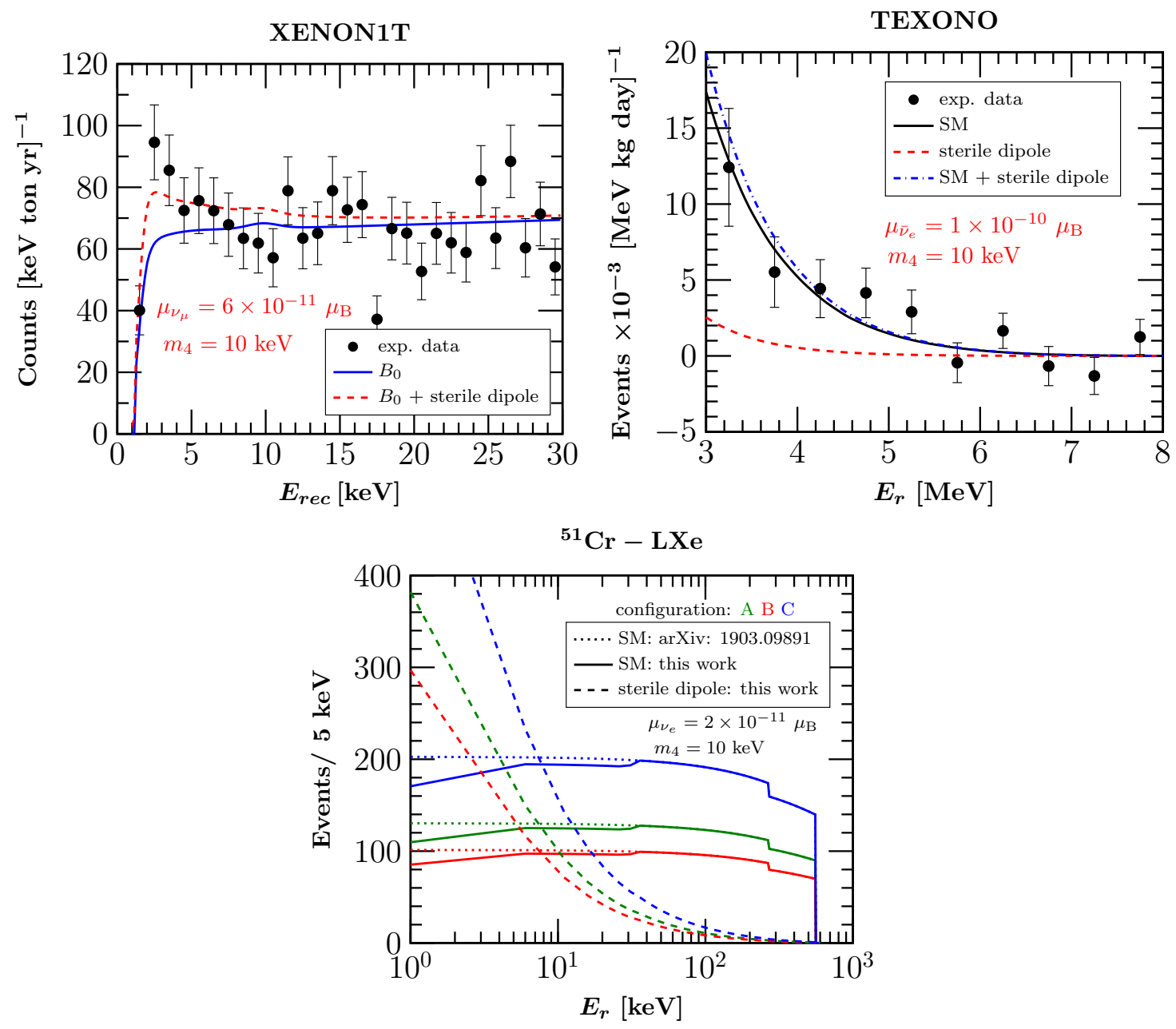

Figure 1. Signal at XENON1T (top left) assuming $\mu_{\nu_{\mu}}=6 \times 10^{-11} \mu_{\mathrm{B}}{ }^{51}$, TEXONO (top right) assuming $\mu_{\bar{\nu}_{e}}=1 \times 10^{-10} \mu_{\mathrm{B}}$ and Cr-LXe (bottom) assuming $\mu_{\nu_{e}}=2 \times 10^{-11} \mu_{\mathrm{B}}$. All cases correspond to a sterile neutrino with mass $m_{4}=10 \mathrm{keV}$.

\section{Results}

Here we present the results of the analysis described in the previous section. We have studied the sensitivity of current and future $\mathrm{CE} \nu \mathrm{NS}$ and $\mathrm{E} \nu \mathrm{ES}$ experiments to the socalled dipole neutrino portal.

Focusing first on the E $\nu$ ES experiments, we concentrate on the solar, reactor, and ${ }^{51} \mathrm{Cr}$ neutrino fluxes, relevant for the XENON1T, TEXONO, and ${ }^{51} \mathrm{Cr}-\mathrm{LX}$ experiments, respectively. For the aforementioned experiments, we illustrate in figure 1 the effect of a neutrino dipole moment assuming the production of a $10 \mathrm{keV}$ sterile neutrino. In particular, the recent XENON1T excess is shown in the top left panel of figure 1, where we present our results assuming the case of a $\nu_{\mu}$ (see ref. [26] for the $\nu_{\tau}$ coupling) with the indicated benchmark value of effective neutrino magnetic moment and sterile neutrino mass. Although there are small differences with the corresponding results of ref. [25], they are 
understandable because the authors did not consider the effect of the step function. Still, their analysis is consistent with the present one, because most of the corrections from the step function are washed out by the XENON1T efficiency. Since the effect of the step function is more pronounced for low threshold experiments, one should stress its importance for studies involving spectral features at low-energy recoils. In the top right panel in figure 1 we show the case of TEXONO, where the reported data points are plotted and compared with the sterile neutrino dipole moment expected signal. Although the effect is visible for these parameters, the statistical uncertainties are still large in this kind of measurements. Finally, in the bottom panel of figure 1 we show the number of events expected in the SM, as well as in the sterile dipole portal, assuming the three different configurations (A, B, C) of the ${ }^{51} \mathrm{Cr}$-LXe detector described previously. Comparing our SM prediction with other reported studies, such as the one in ref. [39], we find they essentially agree, except for the extra corrections associated to bound electrons incorporated in our present work.

We turn now our attention to the CE $\nu$ NS case. The upper panel of figure 2 illustrates the expected signal assuming a magnetic moment of $\mu_{\nu_{\mu}}=2 \times 10^{-9} \mu_{B}$ and a sterile neutrino mass $m_{4}=10 \mathrm{keV}$ at the CsI (left) and LAr (right) detector subsystems of COHERENT. ${ }^{8}$ In the lower panel, we present the corresponding result for the proposed ${ }^{51} \mathrm{Cr} \mathrm{CE} \nu \mathrm{NS}$ experiment described above, assuming $\mu_{\nu_{e}}=2 \times 10^{-11} \mu_{B}$ and various detector materials. Notice that, for the case of the proposed ${ }^{51} \mathrm{Cr}$-type experiment, we have used a neutrino magnetic moment strength that is two orders of magnitude lower compared to the assumed value for COHERENT. This is possible thanks to the extremely low detection threshold achievable with these future detectors. However, a ${ }^{51} \mathrm{Cr}$ neutrino source experiment will be restricted to sub-MeV neutrino masses, while for the higher energy $\pi$-DAR neutrinos at COHERENT, the sensitivity holds up to masses $m_{4} \lesssim m_{\mu} / 2 \simeq 50 \mathrm{MeV}$.

As a first step, assuming the neutrino magnetic moment as an effective parameter, we perform a sensitivity analysis for all the $\mathrm{CE} \nu \mathrm{NS}$ and $\mathrm{E} \nu \mathrm{ES}$ experiments discussed above. A summary of our results is depicted in figure 3, where we show current limits and projected sensitivities at $90 \%$ C.L. One sees that, for this case, the allowed sterile dipole moment required to account for the XENON1T excess lies in the range $2 \times 10^{-11} \lesssim \mu_{\nu_{\mu}} \lesssim 8 \times$ $10^{-11} \mu_{B}$, for a sterile neutrino mass up to $150 \mathrm{keV}$. Note also that our analysis of the XENON1T excess data for the case of the $\nu_{\mu} \rightarrow \nu_{s}$ transition agrees well with ref. [25] and is shown here for comparison. Also shown, is the corresponding result derived in this work for the $\nu_{e} \rightarrow \nu_{s}$ transition. As expected, the latter is consistent with a lower effective magnetic moment due to higher statistics. Indeed, the low-energy solar neutrino flux arriving at the Earth, to which XENON1T is mostly sensitive, contains more $\nu_{e}$ compared to $\nu_{\mu}$. For the $\nu_{\tau} \rightarrow \nu_{s}$ transition, see ref. [26].

Encouraged by these results, we now proceed with the analysis of CE $\nu \mathrm{NS}$ and $\mathrm{E} \nu \mathrm{ES}$ data in novel experimental setups that could probe the above parameter space. As can be seen in figure 3 , the COHERENT data rules out the region with $\mu_{\nu_{\mu}} \gtrsim 3 \times 10^{-9} \mu_{B}$ and $m_{4} \lesssim 50 \mathrm{MeV}$, with the CsI detector performing slightly better compared to LAr. ${ }^{9}$

\footnotetext{
${ }^{8}$ This value is allowed by the recent analyses of COHERENT-CsI [55] and COHERENT-LAr [63].

${ }^{9}$ Only $\nu_{\mu} \rightarrow \nu_{s}$ transitions are assumed for the analysis of COHERENT data. The new CsI data recently reported by the COHERENT collaboration [64] will modify these constraints. However, since the
} 

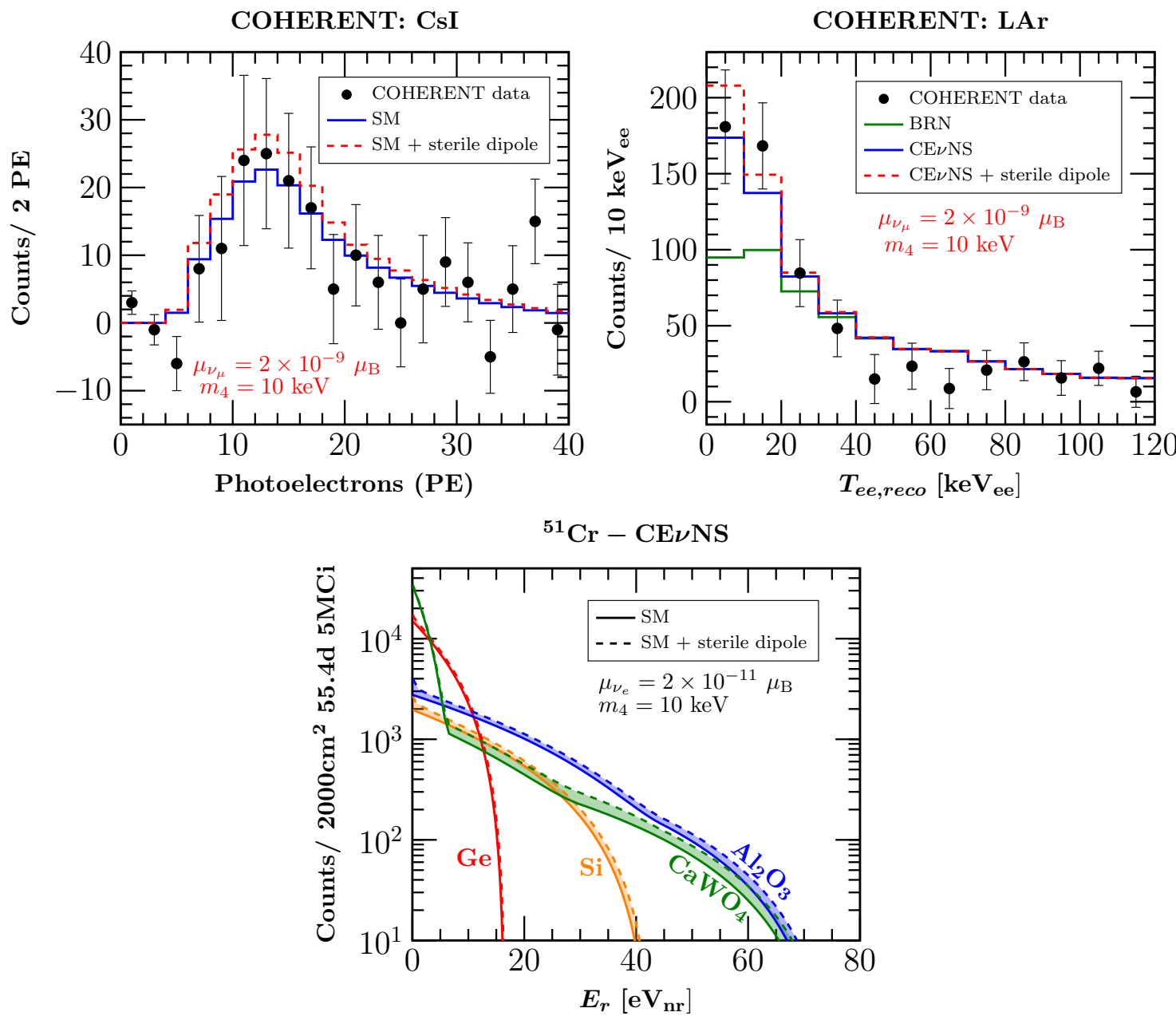

Figure 2. Signal at COHERENT-CsI detector (top left), COHERENT-LAr detector (top right) assuming a transition magnetic moment $\mu_{\nu_{\mu}}=2 \times 10^{-9} \mu_{\mathrm{B}}$ and at a ${ }^{51} \mathrm{Cr} \mathrm{CE} \nu \mathrm{NS}$ experiment (bottom) assuming a transition magnetic moment $\mu_{\nu_{e}}=2 \times 10^{-11} \mu_{\mathrm{B}}$. All cases correspond to a sterile neutrino with mass $m_{4}=10 \mathrm{keV}$.

Concerning the potential of future dipole moment probes, we also show the sensitivity region for the next generation CENNS-750 detector with $610 \mathrm{~kg}$ fiducial mass and 3 years of data aquisition [65]. ${ }^{10}$ For the case of $\mathrm{E} \nu \mathrm{ES}$, the current restrictions are coming from the TEXONO experiment, and one sees how $\mu_{\bar{\nu}_{e}} \gtrsim 3 \times 10^{-10} \mu_{B}$ is excluded, i.e. the constraint is improved by one order of magnitude for the neutrino dipole moment coupling, compared to COHERENT results. However, there is a sharp sensitivity loss at $m_{4} \sim 10 \mathrm{MeV}$, due to the kinematic cut imposed by the low energy of reactor neutrinos.

Finally, we now turn to the expected sensitivities for a ${ }^{51} \mathrm{Cr}$ source neutrino flux. Both for the case of $\mathrm{CE} \nu \mathrm{NS}$ and $\mathrm{E} \nu \mathrm{ES}$ measurements, they would lead to a breakthrough

experimental threshold remains the same as in the previous run, and the TMM signal is governed mainly by the low-energy behavior, we expect that the improvement will be rather mild.

${ }^{10}$ The backgrounds are taken from the estimations of ref. [30]. 


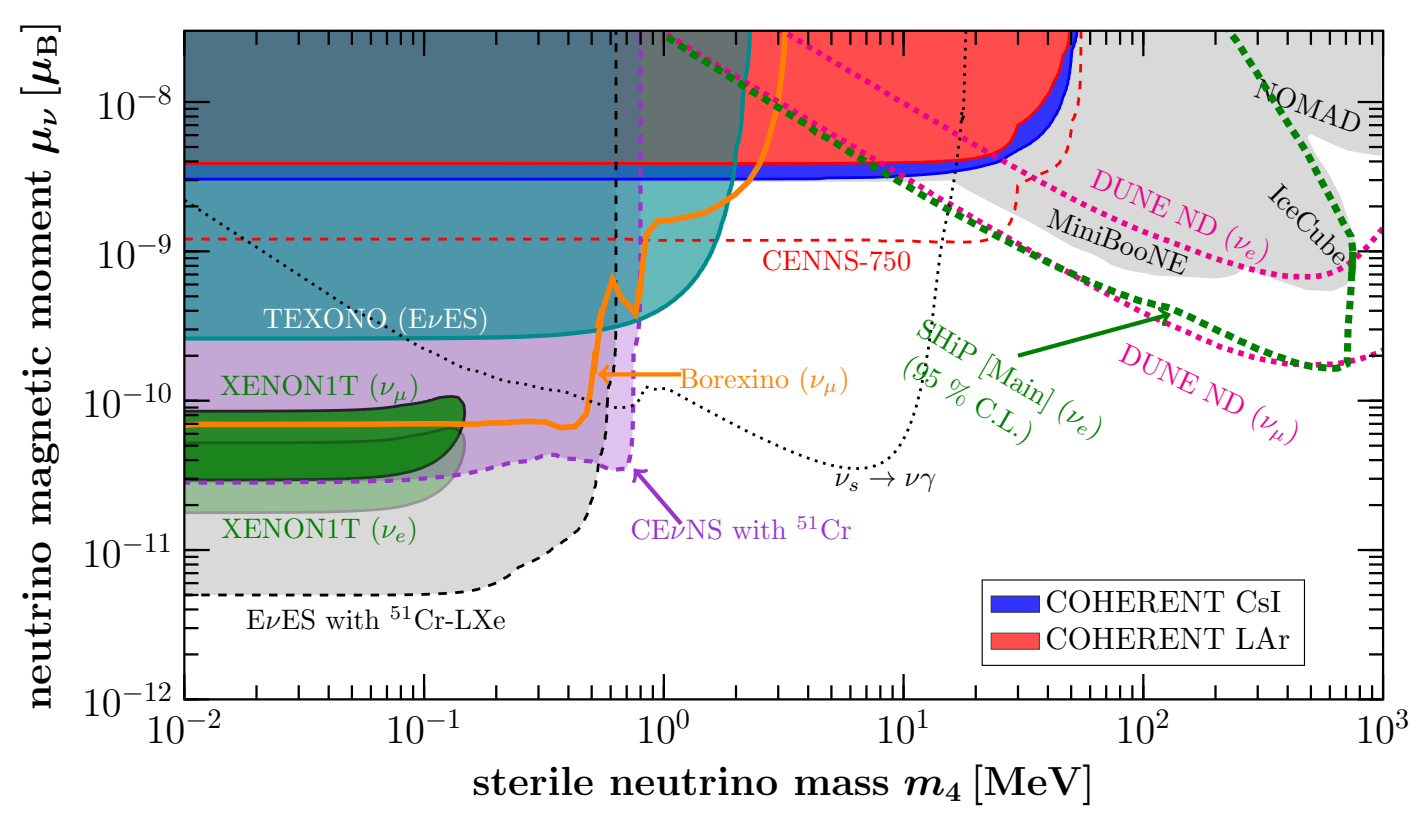

Figure 3. Sensitivity of $\mathrm{CE} \nu \mathrm{NS}$ and $\mathrm{E} \nu \mathrm{ES}$ experiments to the effective sterile neutrino transition magnetic moments. Relevant limits from other experiments are shown for comparison. Solid (dashed) lines correspond to current (future) experiments (see text).

sensitivity reach of a neutrino magnetic moment in the region $\mu_{\nu_{e}} \sim 10^{-12} \mu_{B}$. As in the case of reactor neutrinos, the very low energy of the emitted neutrinos at the ${ }^{51} \mathrm{Cr}$ decay leads to a loss of sensitivity for $m_{4} \gtrsim 750 \mathrm{keV}$. We also note that the $\mathrm{E} \nu \mathrm{ES}{ }^{51} \mathrm{Cr}-\mathrm{LXe}$ case was found to be the only experimental setup where the interference cross-section is non-negligible with respect to the purely magnetic one.

Before closing this discussion, we wish to emphasize the complementarity of the bounds derived here from the analysis of $\mathrm{CE} \nu \mathrm{NS}$ and $\mathrm{E} \nu \mathrm{ES}$ experiments, with those from oscillation experiments. ${ }^{11}$ For the case of the $\nu_{\mu} \rightarrow \nu_{s}$ channel in COHERENT, complementary constraints and sensitivities come from MiniBooNE [66], NOMAD [34], IceCube [28], Borexino [25] and DUNE near detector (ND) [67, 68]. One can see that, though not placing severe constraints on the neutrino magnetic moment, the current and future COHERENT data cover a large portion of the previously unexplored parameter space, overlapping with regions already probed by the aforementioned large scale experiments. We should also mention that, although not visible, COHERENT-CsI is competitive to CHARM-II constraints [28], while the latter will be completely overridden by the future CENNS-750 experiment. Finally, also shown is the sensitivity obtained from the $\nu_{s} \rightarrow \nu \gamma$ decay in ref. [69], which is clearly complementary to the CENNS-750 and ${ }^{51} \mathrm{Cr} \mathrm{CE} \nu \mathrm{NS}$ experiments studied here. Similarly, for the case of TEXONO and ${ }^{51} \mathrm{Cr}$-based CE $\nu$ NS and E $\nu \mathrm{ES}$ experiments ( $\nu_{e} \rightarrow \nu_{s}$ transitions), the relevant experiments would be XENON1T, SHiP and DUNE ND. Using this one may compare our results in figure 3 for the proposed Chromium experiments with the relevant sensitivities from SHiP [34] as well as DUNE ND [67, 68].

\footnotetext{
${ }^{11}$ Only limits from laboratory experiments are discussed. For astrophysical limits, see ref. [34].
} 
One sees that there is no overlap with the $\mathrm{CE} \nu \mathrm{NS}$ and $\mathrm{E} \nu \mathrm{ES}$ experiments. In contrast, the ${ }^{51} \mathrm{Cr}$-LXe setup discussed here can provide an independent test of the region indicated by the XENON1T excess. ${ }^{12}$ However, we emphasize that one should really use eq. (2.5) for the effective magnetic moment for solar neutrino experiments. Only such general $\lambda$-formalism provides a basis for making such comparisons.

Having presented our results for effective neutrino magnetic moments, we now explore the current and future sensitivities of the aforementioned $\mathrm{CE} \nu \mathrm{NS}$ and $\mathrm{E} \nu \mathrm{ES}$ experiments for the TMMs, as expressed within the general formalism discussed in section 2. As emphasized at the Introduction, in contrast to the simple effective magnetic moment description, the adoption of the more general TMMs formalism allows for a direct comparison of the attainable sensitivities at different types of experiments in terms of the same fundamental parameters, $\tilde{\lambda}_{i j}$. Moreover, adopting this general formalism allows the full data set of experiments exposed to a neutrino source with multiple flavors to be used in combined analyses. Hence, for COHERENT and XENON1T, we do not need to consider one nonzero effective magnetic moment $\mu_{\nu_{\alpha}}(\alpha=e, \mu, \tau)$ at a time. In what follows, all relevant $\mu_{\nu_{\alpha}}$ will be assumed non-vanishing and will be expressed in terms of the basic TMMs $\lambda_{i j}$. We present our results for a simplified case, assuming only one non-vanishing TMM $\tilde{\lambda}_{i j}$ at a time, and neglecting the associated CP phases (for a discussion on the impact of the $\mathrm{CP}$ phases see ref. [43]). The current constraints and future sensitivities are shown in figure 4, where one sees the same qualitative behavior as in the case of effective dipole moments.

\section{Conclusions and outlook}

Motivated by the XENON1T excess and by the intrinsic interest in probing neutrino electromagnetic properties, we have examined the current and future experimental sensitivities to a dipole portal interaction associated to a massive sterile neutrino with transition magnetic moment. We have explored such scenario for various $\mathrm{CE} \nu \mathrm{NS}$ and $\mathrm{E} \nu \mathrm{ES}$ experimental setups, analyzing their potential in probing the region of interest for the XENON1T excess.

Besides presenting the relevant sensitivities in terms of the usual effective magnetic moments, we have given the first comprehensive description in terms of the fundamental TMM parameters, see tables 1 and 2. Interference between weak and magnetic terms, possible for massive sterile neutrinos, has been found to play no essential role in constraining the parameters. The only exception to this was found when considering very-low energy $\mathrm{E} \nu \mathrm{ES}$ from a ${ }^{51} \mathrm{Cr}$ source.

Our phenomenological analysis has focused on current and future $\mathrm{CE} \nu \mathrm{NS}$ and $\mathrm{E} \nu \mathrm{ES}$ experiments using low-energy neutrinos from artificial neutrino sources, such as reactors and accelerators, as well as those emerging from a ${ }^{51} \mathrm{Cr}$ source. Our analysis shows that the current constraints arising from the recent COHERENT CE $\nu$ NS measurements on CsI and LAr, as well as from reactor neutrino E $\nu$ ES measurements by TEXONO, can cover a wider, previously unexplored, region in sterile neutrino parameters. In particular, we have shown that the proposed ${ }^{51} \mathrm{Cr}$ experiments can fully probe the explanation of the XENON1T anomaly with the sterile dipole portal (see figures 3 and 4). Finally, we have

\footnotetext{
${ }^{12}$ Bounds from existing nuclear recoil XENON1T data [70] and future LHC projections [71] are weaker.
} 

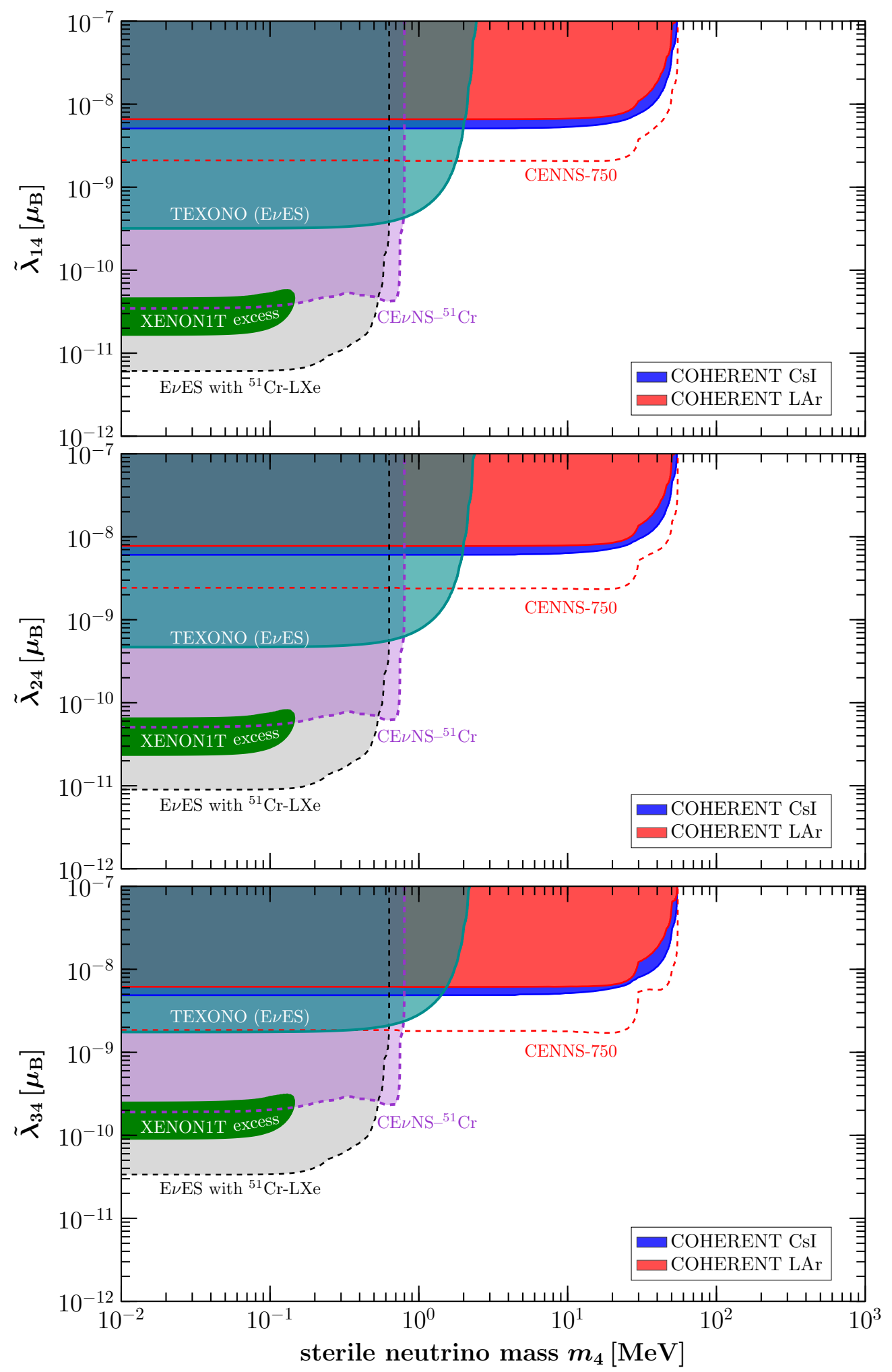

Figure 4. Sensitivity of $\mathrm{CE} \nu \mathrm{NS}$ and $\mathrm{E} \nu \mathrm{ES}$ experiments in terms of the general TMM description. Note that our TMM notation provides a common basis for describing all possible experimental setups, see text. 
also emphasized the complementarity of future low-energy $\mathrm{CE} \nu \mathrm{NS}$ and $\mathrm{E} \nu \mathrm{ES}$ experiments with large-scale experiments, such as DUNE ND, SHiP, Borexino, MiniBooNE, IceCube, and NOMAD, as seen in section 4 .

\section{Acknowledgments}

Work supported by the Spanish grants PID2020-113775GB-I00 (AEI/10.13039/501100011033) and PROMETEO/2018/165 (Generalitat Valenciana), by Fundacão para a Ciência e a Tecnologia (FCT, Portugal) through grant CERN/FIS-PAR/0004/2019 and by CONACYTMexico under grant A1-S-23238. O.G.M. has been supported by SNI (Sistema Nacional de Investigadores). The work of DKP is co-financed by Greece and the European Union (European Social Fund-ESF) through the Operational Programme "Human Resources Development, Education and Lifelong Learning" in the context of the project "Reinforcement of Postdoctoral Researchers - 2nd Cycle" (MIS-5033021), implemented by the State Scholarships Foundation (IKY).

Open Access. This article is distributed under the terms of the Creative Commons Attribution License (CC-BY 4.0), which permits any use, distribution and reproduction in any medium, provided the original author(s) and source are credited.

\section{References}

[1] I. Antoniadis, N. Arkani-Hamed, S. Dimopoulos and G.R. Dvali, New dimensions at a millimeter to a Fermi and superstrings at a TeV, Phys. Lett. B 436 (1998) 257 [hep-ph/9804398] [INSPIRE].

[2] A. Ioannisian and J.W.F. Valle, Light sterile neutrino from extra dimensions and four neutrino solutions to neutrino anomalies, Phys. Rev. D 63 (2001) 073002 [hep-ph/9911349] [INSPIRE].

[3] P. Chen, G.-J. Ding, A.D. Rojas, C.A. Vaquera-Araujo and J.W.F. Valle, Warped flavor symmetry predictions for neutrino physics, JHEP 01 (2016) 007 [arXiv:1509.06683] [INSPIRE].

[4] J.T. Peltoniemi, D. Tommasini and J.W.F. Valle, Reconciling dark matter and solar neutrinos, Phys. Lett. B 298 (1993) 383 [INSPIRE].

[5] J.T. Peltoniemi and J.W.F. Valle, Reconciling dark matter, solar and atmospheric neutrinos, Nucl. Phys. B 406 (1993) 409 [hep-ph/9302316] [INSPIRE].

[6] T.J. Allen, R. Johnson, S. Ranfone, J. Schechter and J.W.F. Valle, Simpson's neutrino and the singular seesaw, Mod. Phys. Lett. A 6 (1991) 1967 [InSPIRE].

[7] M. Drewes et al., A White Paper on keV Sterile Neutrino Dark Matter, JCAP 01 (2017) 025 [arXiv: 1602.04816] [INSPIRE].

[8] C. Giunti and T. Lasserre, eV-scale Sterile Neutrinos, Ann. Rev. Nucl. Part. Sci. 69 (2019) 163 [arXiv: 1901.08330] [inSPIRE].

[9] B. Dasgupta and J. Kopp, Sterile Neutrinos, Phys. Rept. 928 (2021) 63 [arXiv:2106. 05913] [INSPIRE]. 
[10] ArgoneuT collaboration, New Constraints on Tau-Coupled Heavy Neutral Leptons with Masses $m_{N}=280-970 \mathrm{MeV}$, Phys. Rev. Lett. 127 (2021) 121801 [arXiv:2106.13684] [INSPIRE].

[11] J. Schechter and J.W.F. Valle, Neutrino Masses in $\mathrm{SU}(2) \times \mathrm{U}(1)$ Theories, Phys. Rev. D 22 (1980) 2227 [INSPIRE].

[12] L. Canetti, M. Drewes, T. Frossard and M. Shaposhnikov, Dark Matter, Baryogenesis and Neutrino Oscillations from Right Handed Neutrinos, Phys. Rev. D 87 (2013) 093006 [arXiv: 1208.4607] [INSPIRE].

[13] E.K. Akhmedov, V.A. Rubakov and A.Y. Smirnov, Baryogenesis via neutrino oscillations, Phys. Rev. Lett. 81 (1998) 1359 [hep-ph/9803255] [INSPIRE].

[14] T. Asaka and M. Shaposhnikov, The $\nu M S M$, dark matter and baryon asymmetry of the universe, Phys. Lett. B 620 (2005) 17 [hep-ph/0505013] [inSPIRE].

[15] M. Drewes, Y. Georis and J. Klarić, Mapping the viable parameter space for testable leptogenesis, arXiv:2106.16226 [INSPIRE].

[16] J. Schechter and J.W.F. Valle, Majorana Neutrinos and Magnetic Fields, Phys. Rev. D 24 (1981) 1883 [Erratum ibid. 25 (1982) 283] [INSPIRE].

[17] L.F. Li and F. Wilczek, Physical processes involving Majorana neutrinos, Phys. Rev. D 25 (1982) 143 [INSPIRE].

[18] J.F. Nieves, Electromagnetic Properties of Majorana Neutrinos, Phys. Rev. D 26 (1982) 3152 [INSPIRE].

[19] B. Kayser, Majorana Neutrinos and their Electromagnetic Properties, Phys. Rev. D 26 (1982) 1662 [INSPIRE].

[20] R.E. Shrock, Electromagnetic Properties and Decays of Dirac and Majorana Neutrinos in a General Class of Gauge Theories, Nucl. Phys. B 206 (1982) 359 [InSPIRE].

[21] O.G. Miranda, T.I. Rashba, A.I. Rez and J.W.F. Valle, Constraining the neutrino magnetic moment with anti-neutrinos from the sun, Phys. Rev. Lett. 93 (2004) 051304 [hep-ph/0311014] [INSPIRE].

[22] O.G. Miranda, T.I. Rashba, A.I. Rez and J.W.F. Valle, Enhanced solar anti-neutrino flux in random magnetic fields, Phys. Rev. D 70 (2004) 113002 [hep-ph/0406066] [INSPIRE].

[23] O.G. Miranda, D.K. Papoulias, M. Tórtola and J.W.F. Valle, XENON1T signal from transition neutrino magnetic moments, Phys. Lett. B 808 (2020) 135685 [arXiv:2007.01765] [INSPIRE].

[24] XENON collaboration, Excess electronic recoil events in XENON1T, Phys. Rev. D 102 (2020) 072004 [arXiv : 2006. 09721] [inSPIRE].

[25] V. Brdar, A. Greljo, J. Kopp and T. Opferkuch, The Neutrino Magnetic Moment Portal: Cosmology, Astrophysics, and Direct Detection, JCAP 01 (2021) 039 [arXiv:2007.15563] [INSPIRE].

[26] I.M. Shoemaker, Y.-D. Tsai and J. Wyenberg, An Active-to-Sterile Neutrino Transition Dipole Moment and the XENON1T Excess, arXiv:2007.05513 [INSPIRE].

[27] S. Karmakar and S. Pandey, XENON1T constraints on neutrino non-standard interactions, arXiv:2007.11892 [INSPIRE]. 
[28] P. Coloma, P.A.N. Machado, I. Martinez-Soler and I.M. Shoemaker, Double-Cascade Events from New Physics in IceCube, Phys. Rev. Lett. 119 (2017) 201804 [arXiv:1707.08573] [INSPIRE].

[29] F.J. Escrihuela, D.V. Forero, O.G. Miranda, M. Tortola and J.W.F. Valle, On the description of nonunitary neutrino mixing, Phys. Rev. D 92 (2015) 053009 [Erratum ibid. 93 (2016) 119905] [arXiv: 1503.08879] [InSPIRE].

[30] O.G. Miranda, D.K. Papoulias, O. Sanders, M. Tórtola and J.W.F. Valle, Future CEvNS experiments as probes of lepton unitarity and light-sterile neutrinos, Phys. Rev. D 102 (2020) 113014 [arXiv : 2008. 02759] [INSPIRE].

[31] D.V. Forero, C. Giunti, C.A. Ternes and M. Tortola, Nonunitary neutrino mixing in short and long-baseline experiments, Phys. Rev. D 104 (2021) 075030 [arXiv:2103.01998] [INSPIRE].

[32] T.S. Kosmas, D.K. Papoulias, M. Tortola and J.W.F. Valle, Probing light sterile neutrino signatures at reactor and Spallation Neutron Source neutrino experiments, Phys. Rev. D 96 (2017) 063013 [arXiv:1703.00054] [INSPIRE].

[33] D. McKeen and M. Pospelov, Muon Capture Constraints on Sterile Neutrino Properties, Phys. Rev. D 82 (2010) 113018 [arXiv:1011.3046] [INSPIRE].

[34] G. Magill, R. Plestid, M. Pospelov and Y.-D. Tsai, Dipole Portal to Heavy Neutral Leptons, Phys. Rev. D 98 (2018) 115015 [arXiv:1803.03262] [INSPIRE].

[35] COHERENT collaboration, Observation of Coherent Elastic Neutrino-Nucleus Scattering, Science 357 (2017) 1123 [arXiv: 1708.01294] [INSPIRE].

[36] COHERENT collaboration, First Measurement of Coherent Elastic Neutrino-Nucleus Scattering on Argon, Phys. Rev. Lett. 126 (2021) 012002 [arXiv: 2003.10630] [INSPIRE].

[37] TEXONO collaboration, Measurement of $\bar{\nu}_{e}$-Electron Scattering Cross-Section with a CsI(Tl) Scintillating Crystal Array at the Kuo-Sheng Nuclear Power Reactor, Phys. Rev. D 81 (2010) 072001 [arXiv:0911.1597] [INSPIRE].

[38] C. Bellenghi, D. Chiesa, L. Di Noto, M. Pallavicini, E. Previtali and M. Vignati, Coherent elastic nuclear scattering of ${ }^{51}$ Cr neutrinos, Eur. Phys. J. C 79 (2019) 727 [arXiv: 1905.10611] [INSPIRE].

[39] J.M. Link and X.-J. Xu, Searching for BSM neutrino interactions in dark matter detectors, JHEP 08 (2019) 004 [arXiv: 1903.09891] [INSPIRE].

[40] P. Vogel and J. Engel, Neutrino Electromagnetic Form-Factors, Phys. Rev. D 39 (1989) 3378 [INSPIRE].

[41] J.F. Beacom and P. Vogel, Neutrino magnetic moments, flavor mixing, and the Super-Kamiokande solar data, Phys. Rev. Lett. 83 (1999) 5222 [hep-ph/9907383] [INSPIRE].

[42] W. Grimus, M. Maltoni, T. Schwetz, M.A. Tortola and J.W.F. Valle, Constraining Majorana neutrino electromagnetic properties from the LMA-MSW solution of the solar neutrino problem, Nucl. Phys. B 648 (2003) 376 [hep-ph/0208132] [INSPIRE].

[43] O.G. Miranda, D.K. Papoulias, M. Tórtola and J.W.F. Valle, Probing neutrino transition magnetic moments with coherent elastic neutrino-nucleus scattering, JHEP 07 (2019) 103 [arXiv: 1905.03750] [INSPIRE]. 
[44] W. Grimus and T. Schwetz, Elastic neutrino electron scattering of solar neutrinos and potential effects of magnetic and electric dipole moments, Nucl. Phys. B 587 (2000) 45 [hep-ph/0006028] [INSPIRE].

[45] J. Barranco, O.G. Miranda and T.I. Rashba, Probing new physics with coherent neutrino scattering off nuclei, JHEP 12 (2005) 021 [hep-ph/0508299] [INSPIRE].

[46] D.K. Papoulias and T.S. Kosmas, Standard and Nonstandard Neutrino-Nucleus Reactions Cross Sections and Event Rates to Neutrino Detection Experiments, Adv. High Energy Phys. 2015 (2015) 763648 [arXiv: 1502.02928] [INSPIRE].

[47] D.K. Papoulias, T.S. Kosmas, R. Sahu, V.K.B. Kota and M. Hota, Constraining nuclear physics parameters with current and future COHERENT data, Phys. Lett. B 800 (2020) 135133 [arXiv: 1903.03722] [INSPIRE].

[48] S. Klein and J. Nystrand, Exclusive vector meson production in relativistic heavy ion collisions, Phys. Rev. C 60 (1999) 014903 [hep-ph/9902259] [INSPIRE].

[49] J.-W. Chen, H.-C. Chi, C.P. Liu and C.-P. Wu, Low-energy electronic recoil in xenon detectors by solar neutrinos, Phys. Lett. B 774 (2017) 656 [arXiv:1610.04177] [INSPIRE].

[50] W. Grimus and P. Stöckinger, Effects of neutrino oscillations and neutrino magnetic moments on elastic neutrino-electron scattering, Phys. Rev. D 57 (1998) 1762 [hep-ph/9708279] [INSPIRE].

[51] D. Baxter et al., Recommended conventions for reporting results from direct dark matter searches, Eur. Phys. J. C 81 (2021) 907 [arXiv:2105.00599] [InSPIRE].

[52] C. Boehm, D.G. Cerdeno, M. Fairbairn, P.A.N. Machado and A.C. Vincent, Light new physics in XENON1T, Phys. Rev. D 102 (2020) 115013 [arXiv: 2006.11250] [INSPIRE].

[53] W.C. Louis, Searches for muon-to-electron (anti) neutrino flavor change, Prog. Part. Nucl. Phys. 63 (2009) 51 [INSPIRE].

[54] A. Konovalov, COHERENT at SNS and CsI[Na] effort update, talk at Magnificent CEvNS, Cyberspace, 16-20 November 2020 and online at https://indico.cern.ch/event/943069/contributions/4066385/.

[55] D.K. Papoulias, COHERENT constraints after the COHERENT-2020 quenching factor measurement, Phys. Rev. D 102 (2020) 113004 [arXiv:1907.11644] [INSPIRE].

[56] COHERENT collaboration, COHERENT collaboration data release from the first observation of coherent elastic neutrino-nucleus scattering, arXiv:1804.09459 [INSPIRE].

[57] COHERENT collaboration, COHERENT collaboration data release from the first detection of coherent elastic neutrino-nucleus scattering on argon, arXiv:2006.12659 [INSPIRE].

[58] M. Cadeddu, F. Dordei, C. Giunti, Y.F. Li, E. Picciau and Y.Y. Zhang, Physics results from the first COHERENT observation of coherent elastic neutrino-nucleus scattering in argon and their combination with cesium-iodide data, Phys. Rev. D 102 (2020) 015030 [arXiv : 2005. 01645] [INSPIRE].

[59] G. Mention et al., The Reactor Antineutrino Anomaly, Phys. Rev. D 83 (2011) 073006 [arXiv: 1101.2755] [INSPIRE].

[60] V.I. Kopeikin, L.A. Mikaelyan and V.V. Sinev, Spectrum of electronic reactor anti-neutrinos, Phys. Atom. Nucl. 60 (1997) 172 [Yad. Fiz. 60 (1997) 230] [InSPIRE]. 
[61] O.G. Miranda, D.K. Papoulias, M. Tórtola and J.W.F. Valle, Probing new neutral gauge bosons with CELNS and neutrino-electron scattering, Phys. Rev. D 101 (2020) 073005 [arXiv: 2002.01482] [INSPIRE].

[62] P. Coloma, P. Huber and J.M. Link, Combining dark matter detectors and electron-capture sources to hunt for new physics in the neutrino sector, JHEP 11 (2014) 042 [arXiv: 1406.4914] [INSPIRE].

[63] O.G. Miranda, D.K. Papoulias, G. Sanchez Garcia, O. Sanders, M. Tórtola and J.W.F. Valle, Implications of the first detection of coherent elastic neutrino-nucleus scattering (CEvNS) with Liquid Argon, JHEP 05 (2020) 130 [Erratum JHEP 01 (2021) 067] [arXiv:2003.12050] [INSPIRE].

[64] D. Akimov et al., Measurement of the Coherent Elastic Neutrino-Nucleus Scattering Cross Section on CSI by COHERENT, arXiv:2110.07730 [INSPIRE].

[65] COHERENT collaboration, Sensitivity of the COHERENT Experiment to Accelerator-Produced Dark Matter, Phys. Rev. D 102 (2020) 052007 [arXiv:1911.06422] [INSPIRE].

[66] MiniBoonE collaboration, A Search for Electron Neutrino Appearance at the $\Delta m^{2} \sim 1 \mathrm{eV}^{2}$ Scale, Phys. Rev. Lett. 98 (2007) 231801 [arXiv:0704.1500] [INSPIRE].

[67] T. Schwetz, A. Zhou and J.-Y. Zhu, Constraining active-sterile neutrino transition magnetic moments at DUNE near and far detectors, JHEP 07 (2021) 200 [arXiv:2105.09699] [INSPIRE].

[68] M. Atkinson, P. Coloma, I. Martinez-Soler, N. Rocco and I.M. Shoemaker, Heavy Neutrino searches through Double-Bang Events at Super-Kamiokande, DUNE, and Hyper-Kamiokande, arXiv:2105.09357 [INSPIRE].

[69] R. Plestid, Luminous solar neutrinos I: Dipole portals, arXiv:2010.04193 [INSPIRE].

[70] I.M. Shoemaker and J. Wyenberg, Direct Detection Experiments at the Neutrino Dipole Portal Frontier, Phys. Rev. D 99 (2019) 075010 [arXiv:1811.12435] [InSPIRE].

[71] A. Ismail, S. Jana and R.M. Abraham, Neutrino Up-scattering via the Dipole Portal at Forward LHC Detectors, arXiv:2109.05032 [INSPIRE]. 